\title{
Biodiversidad marina de Costa Rica: Crustacea: Infraorden Anomura
}

\author{
Rita $\operatorname{Vargas}^{1} \&$ Jorge Cortés ${ }^{1,2}$ \\ 1 Museo de Zoología, Escuela de Biología, Universidad de Costa Rica, San Pedro, San José 2060, Costa Rica. Fax (506) \\ 207-4216; ritav@biologia.ucr.ac.cr \\ 2 Centro de Investigación en Ciencias del Mar y Limnología (CIMAR), Universidad de Costa Rica, San Pedro, San José \\ 2060, Costa Rica.
}

Recibido 27-VI-2002. Corregido 28-I-2004. Aceptado 25-XI-2004.

\begin{abstract}
Marine biodiversity of Costa Rica: Crustacea: Infraorder Anomura. The anomuran crabs are among the best known crustacean groups from the Pacific coast. However, this group is poorly known from the Caribbean coast of Costa Rica. In this compilation based on the literature and the collection at the Zoology Museum, Biology School, University of Costa Rica, we report the presence of 114 species of the Infraorder Anomura for Costa Rica, 20 species from the Caribbean, 96 species from the Pacific (two are present on both coasts). Twenty-nine species are new reports for Costa Rica, 15 from the Caribbean coast (74\% of the total of species from that coast) and 14 from the Pacific (15\% of the total from the Pacific). The range of ten species is extended to Costa Rica, siete from the Caribbean and three from the Pacific. Six species are reported for the first time from Cocos Island, where there are also four endemic species. Rev. Biol. Trop. 54(2): 461-488. Epub 2006 Jun 01.
\end{abstract}

Key words: Anomura, Crustacea, crabs, biodiversity, Costa Rica.

Los cangrejos anomuros constituyen uno de los grupos de crustáceos más estudiados en el Pacífico Este Tropical que incluye Costa Rica (Faxon 1893, 1895, Haig 1960, Effort 1971, 1973, 1976, Ball y Haig 1974, Gore 1982, Hendrickx y Harvey 1999). Algunos otros trabajos en decápodos incluyen información sobre los anomuros en esta región (Lemaitre y Alvarez-León 1992, Morán y Dittel 1993). En el Caribe también ha sido un grupo muy estudiado pero curiosamente esto no es así para el Caribe de Centroamérica, exceptuando a Panamá en cuya costa Caribe se han realizado varias investigaciones (Chace 1942, Gore 1970, Gore y Abele 1976, Abele y Kim 1989, Campos y Lemaitre 1994).

El propósito de este trabajo es recopilar la información existente sobre anomuros en Costa Rica, basado tanto en la literatura como también en las colecciones del Museo de
Zoología, Escuela de Biología, Universidad de Costa Rica. Otro objetivo es motivar, a través de esta recopilación, futuras investigaciones sobre este importante grupo de crustáceos sobre todo en la costa Caribe de Costa Rica.

\section{MATERIAL Y MÉTODOS}

Para el presente estudio se contempló solamente las especies informadas en la literatura para Costa Rica y las que están depositadas en el Museo de Zoología. La mayoría de los especímenes fueron preservados primero en formalina al $10 \%$ y posteriormente almacenados en alcohol de 70\%. El material está catalogado bajo las siglas UCR y el número correspondiente. La colección de Anomuros está debidamente corroborada por especialistas en el campo. Se siguió la clasificación propuesta por 
Martin y Davis (2001) para el ordenamiento de las familias y se colocaron los géneros y especies alfabéticamente. Para cada especie se da información acerca del material presente en las colecciones, el hábitat y la profundidad mencionada en otras publicaciones o de la etiqueta del espécimen, informes previos para el país, y su distribución conocida.

Las abreviaciones utilizadas son: UCR, Universidad de Costa Rica; CR, Costa Rica; s.f., sin fecha; ov., hembras con huevos; juv., juveniles.

\section{RESULTADOS}

\section{Costa Caribe}

Infraorden Anomura H. Milne Edwards, 1822

Superfamilia Galatheoidea Samouelle, 1819

Familia GALATHEIDAE Samouelle, 1819

\section{Munida flinti \\ Benedict, 1902}

Material: 19 ov., $3.2 \mathrm{~km}$ al norte de Limón, 1.IV.1968 (UCR 123-01).

Hábitat: 110-203 m (Benedict 1902).

Informes previos en CR: ninguno.

Distribución: golfo de México (Benedict 1902), al norte del arrecife Alacrán y mar afuera de Grenada (Chace 1942).

Familia PORCELLANIDAE Haworth, 1825

\section{Megalobrachium mortenseni}

Haig, 1962

Material: $1 \sigma^{\top} 1 \%$, puerto Vargas, Parque Nacional Cahuita, Limón, 30.X.1988 (UCR 1729-01). $20^{\top}$, puerto Vargas, Parque Nacional Cahuita, Limón, 24.IV.1998 (UCR 2224-01).

Hábitat: debajo de piedras superpuestas sobre cascajo y arena gruesa (Werding 1977). En hoyos y grietas de rocas coralinas cubiertas por algas y esponjas a $1.5 \mathrm{~m}$ de profundidad en la laguna del arrecife (este estudio).

Informes previos en CR: ninguno.
Distribución: islas Vírgenes, Panamá, costa Caribe de Colombia a Brasil (Gore 1982).

\section{Megalobrachium poeyi \\ (Guérin, 1855)}

Material: $1 \mathcal{+}$, puerto Vargas, Parque Nacional Cahuita, Limón, 24.IV.1998 (UCR 2224-04).

Hábitat: en acumulaciones de rocas o coral muerto en áreas someras y protegidas, también en praderas de Thalassia (Werding 1984).

Informes previos en CR: ninguno.

Distribución: Florida, Antillas Mayores y Menores, costa norte de Suramérica y Panamá (Werding 1984).

\section{Megalobrachium roseum \\ (Rathbun, 1900)}

Material: $21 \overbrace{}^{\nearrow} 15 \bigcirc, 4$ juv., puerto Vargas, Parque Nacional Cahuita, Limón, 24.IV.1998 (UCR 2224-02).

Hábitat: grietas pequeñas entre piedras superpuestas, las bases de los gorgonáceos y sobre animales sésiles (Werding 1977). En el litoral (Gore y Abele 1976). En hoyos y grietas de rocas coralinas cubiertas por algas y esponjas a $1.5 \mathrm{~m}$ de profundidad en la laguna del arrecife (este estudio).

Informes previos en CR: ninguno.

Distribución: costa continental de Sur y Centroamérica, incluyendo Panamá, Colombia, Venezuela y Brasil (Werding 1977).

\section{Megalobrachium soriatum}

(Say, 1818)

Material: $10^{\top}$, puerto Vargas, Parque Nacional Cahuita, Limón, 23.IX.1995 (UCR 2219-02).

Hábitat: según Werding (1977) está especie ocupa el mismo hábitat que $M$. roseum

Informes previos en CR: ninguno.

Distribución: desde Carolina del Norte, Florida y Golfo de México, Panamá, Colombia y de Barbados a Brasil (Werding 1977). 
Minyocerus angustus

Diana, 1852

Material: ninguno.

Hábitat: es comensal de estrellas de mar de los géneros Luidia, Tethyaster y Astropecten, recolectado hasta $29 \mathrm{~m}$ (Gore 1974).

Informes previos en CR: frente a Tortuguero, Limón (Gore 1974).

Distribución: desde Bluefields, Nicaragua al sur hasta Desterro, Brasil (Gore 1974).

\section{Pachycheles cristobalensis Gore, 1970}

Material: $20^{\top} 1 \bigcirc$ ov., 1 juv., punta Mona, Limón, 1.X.1999 (UCR 2305-01).

Hábitat: en la zona entre mareas, recolectados utilizando Pronoxfish y una red de mano, sobre una plataforma rocosa donde se presentan algas como Sargassum, Caulerpa, Halimeda y otras (Gore 1970). Asociados con el coral Siderastrea siderea entre 1-3 m (J. Cortés).

Informes previos en CR: ninguno.

Distribución: conocido hasta ahora solo de una franja alrededor de la entrada en el lado Caribe del Canal de Panamá (Gore y Abele 1976).

Pachycheles pilosus

(H. Milne Edwards, 1837)

Material: $1{ }^{\top}$, punta Uva, Limón, 1.X.1999 (UCR 2304-03).

Hábitat: grietas en rocas o pedazos de coral muerto y en conglomerados pegados por esponjas (Werding 1984). Asociados con el coral Siderastrea siderea entre 1-3 m (J. Cortés).

Informes previos en CR: ninguno.

Distribución: Carolina del Sur, Bahamas, varios lugares entre Puerto Rico y Tobago, las Antillas Holandesas, Santa Marta y Cartagena en la costa continental de América del Sur, isla Providencia (Werding 1984).

\section{Pachycheles serratus}

(Benedict, 1901)

Material: $10^{\top} 2$ ( 1 ov.), entre Manzanillo y punta Uva, Limón, 12.XI.1988 (UCR 153401). $1 \overbrace{}^{\text {T } 1} 1$ ov ov., puerto Vargas, Parque Nacional Cahuita, Limón, 24.IV.1998 (UCR 2224-15).

Hábitat: común en intersticios y grietas de rocas coralinas (Gore y Abele 1976) del litoral a 6 m (Gore 1982).

Informes previos en CR: ninguno.

Distribución: Antillas, Panamá y Colombia (Gore 1982).

Comentario: se amplía la distribución al norte hasta puerto Vargas, Parque Nacional Cahuita, Limón, Costa Rica.

\section{Petrolisthes armatus \\ (Gibbes, 1850)}

Material: $10^{\pi}$, puerto Vargas, Cahuita, 30.X.1988 (UCR 1659-01).

Hábitat: en la zona entre mareas bajo rocas en bancos de ostras y mejillones, alrededor de manglares, en corales y esponjas, desde el litoral hasta $30 \mathrm{~m}$ (Haig 1960).

Informes previos en CR: bahía Salinas y Puntarenas (Haig 1960), bahías Santa Elena y Culebra, isla Tortuga y bahía Coronado (Haig 1968).

Distribución: en el Atlántico desde África Occidental hasta Florida y en el Caribe hasta Brasil; En el Pacífico del golfo de California a Perú (Haig 1960).

Comentario: nuevo registro para el Caribe de Costa Rica.

\section{Petrolisthes galathinus}

(Bosc, 1801)

Material: 19 ov., Limón, 25.VII.1964 (UCR 65-01). $50^{\text {T }} 6$ \% , puerto Vargas, Parque Nacional Cahuita, Limón, 24.IV.1998 (UCR 2224-03). $10^{\top} 1$, entre Manzanillo y punta Uva, 12.XI.1988 (UCR 1534-02). 
Hábitat: en el Atlántico, bajo rocas y asociado con esponjas, corales y anémonas. En el Pacífico asociado a fondos de arena y arena con conchas (Haig 1960). Desde el litoral hasta $54 \mathrm{~m}$ (Gore 1982).

Informes previos en CR: isla San Lucas, golfo de Nicoya (Haig 1960); playas del Coco (Gore 1982).

Distribución: en el Atlántico en cabo Hatteras, golfo de México y del Mar Caribe hasta Brasil. En el Pacífico de Costa Rica a Ecuador (Gore 1982).

Comentario: nuevo registro para el Caribe de Costa Rica.

\section{Petrolisthes marginatus \\ Stimpson, 1858}

Material: 19 ov., isla Uvita, 3.X.1970 (UCR 884-01).

Hábitat: litoral, bajo rocas y colonias de coral (Haig 1956).

Informes previos en CR: ninguno.

Distribución: África Occidental, isla Ascensión, en el sur del mar Caribe desde Barbados a Panamá, la costa norte de Sur América y Brasil (Gore 1982).

Comentarios: Las citas de P. marginatus para la costa del Pacífico americano corresponden a $P$. haigae Chace, 1962 (vease infra).

\section{Porcellana sayana \\ (Leach, 1820)}

Material: $20^{\top} 3 q, 1$ juv., $3 \mathrm{~km}$ al norte de Manzanillo, Limón, 20.X.1998 (UCR 2302-01).

Hábitat: comensal obligado sobre otros animales (Werding 1984). Los especímenes arriba mencionados fueron recolectados como comensales del ermitaño Petrochirus diogenes a $23 \mathrm{~m}$.

Informes previos en CR: ninguno.

Distribución: de Carolina del Norte al estado de Río Grande do Sul, Brasil incluyendo golfo de México, Florida, Bahamas, el Caribe y las Antillas (Werding 1984).

Comentario: primer informe para Costa Rica.
Superfamilia Hippoidea Latreille, 1825

Familia ALBUNEIDAE Stimpson, 1858

Albunea paretii

Guérim-Meneville, 1853

Material: $10^{\top}$, isla Uvita, Limón, 4.X.1970 (UCR 1666-01).

Hábitat: fondos arenosos, de la línea inferior de marea a $38 \mathrm{~m}$ (Williams 1984).

Informes previos en CR: ninguno.

Distribución: de Carolina del Norte a Corpus Christi, Texas; a través de las Antillas Mayores a Santa Catarina, Brasil; en África Occidental de las islas Cabo Verde a Senegal y Ghana (Williams 1984).

\section{Lepidopa richmondi Benedict, 1903}

Material: ninguno.

Hábitat: en arena en la zona entre mareas (Dexter 1974).

Informes previos en CR: Puerto Viejo, Limón (Dexter 1974).

Distribución: de Nicaragua a Brasil; incluyendo las islas del Caribe (Efford 1971).

Familia HIPPIDAE Latreille, 1825

\section{Emerita brasiliensis \\ Schmitt, 1935}

Material: ninguno.

Hábitat: en arena en la zona de entre mareas (Dexter 1974).

Informes previos en CR: playa Bonita, playa del aeropuerto y Cahuita, Limón (Dexter 1974).

Distribución: Yucatán, México; Trinidad, Venezuela hasta Uruguay (Efford 1976).

\section{Hippa cubensis \\ (de Saussure, 1857)}

Material: 1 ð, 1 juv., playa Juana López, Limón, 8.I.1995 (UCR 2019-01).

Hábitat: en arena en la zona entre mareas (este trabajo). 
Informes previos en CR: Cahuita, Limón (Dexter 1974).

Distribución: de Florida a Brasil; Antillas Mayores, isla Ascensión, Bahamas, África Occidental, Puerto Rico, Hucares y San Tomas (Abele y Kim 1986).

Superfamilia Paguroidea Latreille, 1802

Familia COENOBITIDAE Dana, 1815

$$
\begin{gathered}
\text { Coenobita clypeatus } \\
\text { (Herbst, 1791) }
\end{gathered}
$$

Material: $1 \sigma^{\pi} 1 \%$, isla Uvita, Limón, 3.X.1970 (UCR 852-01). 19, Limón, II.1962 (UCR 1516-01).

Hábitat: En la costa, en bosques y márgenes de manglares (Bright 1966).

Informes previos en CR: Puerto Viejo, Moín, Portete y Cahuita (Bright 1966).

Distribución: Florida, Bermuda, de las Antillas Mayores a Venezuela (Williams 1984).

Familia DIOGENIDAE Ortmann, 1892

$$
\begin{gathered}
\text { Calcinus tibicen } \\
\text { (Herbst, 1790) }
\end{gathered}
$$

Material: $20^{\nearrow} 19$, Puerto Viejo, 14.II,1981 (UCR 1034).

Hábitat: de la parte más baja de la zona entre mareas hasta $33 \mathrm{~m}$ de profundidad, se encuentra comúnmente en sustratos duros como fondos rocosos, plataformas arrecifales de poliquetos y arrecifes de coral (Campos y Lemaitre 1994).

Informes previos en CR: ninguno.

Distribución: Florida, golfo de México, del Caribe a Brasil incluyendo Bermuda (Campos y Lemaitre 1994).

\section{Petrochirus diogenes (Linnaeus, 1758)}

Material: $20^{\top}$, barra del Colorado, Limón,

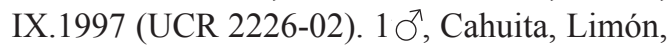

18.X.1968 (UCR 1211-01). 1 $0^{\top}, 3 \mathrm{~km}$ al norte de Manzanillo, Limón, 20.X.1998 (UCR 230202).

Hábitat: lodo, lodo y conchas, fondos arenosos, en parches de Thalassia; entre 18 y $128 \mathrm{~m}$ (Williams 1984).

Informes previos en CR: ninguno.

Distribución: de cabo Lookout, Carolina del Norte, a través del golfo de México y las Antillas Mayores a isla de Sao Sevastiao, Brasil (Abele y Kim 1986).

\section{Costa Pacífica}

Infraorden Anomura H. Milne Edwards, 1822 Superfamilia Galatheoidea Samouelle, 1819 Familia GALATHEIDAE Samouelle, 1819

\section{Munida gracilipes}

Faxon, 1893

Material: $1 \delta^{\top}$, mar adentro de isla Damas,

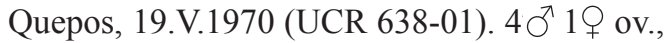
golfo Dulce, 11.XII.1993 (UCR 2005-03). 15 ठ 24 (22 ov.), golfo Dulce, 10.II.1999 (UCR 2006-01).

Hábitat: a 280 m (Faxon 1895).

Informes previos en CR: ninguno.

Distribución: Panamá a Colombia (Lemaitre y Alvarez-León 1992).

\section{Munida obesa \\ Faxon, 1893}

Material: $10^{\top} 2 q$ ov., golfo de Nicoya

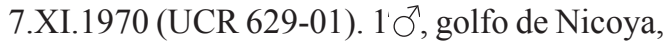
10.XI.1970 (UCR 631-01). 3 9 , mar adentro al sur de isla Negritos, golfo de Nicoya, 8.V.1970 (UCR 637-02).

Hábitat: ha sido capturada en la plataforma y talud continental (Hendrickx 1995). De 333 a 384 m (Faxon 1895).

Informes previos en CR: ninguno.

Distribución: golfo de Panamá (Faxon 1895), Colombia (Lemaitre y Alvarez-León 1992). 
Munida perlata

Benedict, 1902

Material: ninguno.

Hábitat: 1 920-3 292 m (Wicksten 1989).

Informes previos en CR: mar afuera de isla del Coco, informada como M. micropthalma Faxon, 1893 y luego como M. perlata por Moran y Dittel (1993).

Distribución: Galápagos (Benedict 1902), isla del Coco (Moran y Dittel 1993), al sur del golfo de California (Wicksten 1989).

\section{Munida refulgens \\ Faxon, 1893}

Material: $2 q$ ov., playas del Coco, 19.II.1965 (UCR 119-01). $120^{\pi} 1$ \%, de Dominical a Quepos, 28.V.1969 (UCR 635-

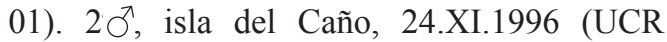
2183-04). $40^{\top}$, isla del Coco, 8.IV.1992 (UCR 2163-01).

Hábitat: 95-183 m (Wicksten 1989).

Informes previos en CR: isla del Coco (Faxon 1895).

Distribución: cerca de las islas Tres Marías, México hasta mar afuera de isla Malpelo, Colombia (Wicksten 1989).

\section{Munidopsis albatrossae}

Pequegnat \& Pequegnat, 1970

Material: ninguno.

Hábitat: $3570 \mathrm{~m}$.

Informes previos en CR: mar afuera de Costa Rica (Pequegnat y Pequegnat 1973)

Distribución: mar afuera de Baja California y Costa Rica (Pequegnat y Pequegnat 1973)

\section{Munidopsis antoni}

(A. Milne Edwards, 1884)

Material: ninguno.

Hábitat: 2 519-3 676 m (Wicksten 1989).

Informes previos en CR: mar afuera de Costa Rica (Wolff 1961) que corresponde a una captura efectuada a $50 \mathrm{~km}$ de la costa.
Distribución: Cosmopolita (Wicksten 1989).

\author{
Munidopsis aspera \\ (Henderson, 1885)
}

Material: ninguno.

Hábitat: 104-2 748 m (Wicksten 1989).

Informes previos en CR: isla del Coco (Faxon 1895).

Distribución: de isla Santa Catalina, California, al estrecho de Magallanes (Wicksten 1989).

\section{Munidopsis ciliata \\ Wood-Mason, 1891}

Material: ninguno.

Hábitat: de $2030-2875 \mathrm{~m}$. (Wicksten 1989), de 1240-1 245 en el talud continental donde convive con una comunidad de crustáceos a lo largo de la cresta en la zona de oxígeno mínimo en el sureste del golfo de California (Hendrickx 2001)

Informes previos en CR: isla del Coco (Faxon 1895).

Distribución: de Oregon y el sureste del golfo de California a Panamá (Wicksten 1989, Hendrickx 2001).

\section{Munidopsis diomedae}

(Faxon, 1893)

Material: ninguno.

Hábitat 768-3 790 m (Wicksten 1989).

Informes previos en CR: isla del Coco (Faxon 1893).

Distribución: de isla San Clemente, California, hasta Chile (Wicksten 1989).

$$
\begin{gathered}
\text { Munidopsis hamata } \\
\text { Faxon, } 1893
\end{gathered}
$$

Material: $10^{\top}$, costa Pacífica, s.f. (UCR 1807-02).

Hábitat: 936-1 337 m (Wicksten 1989).

Informes previos en CR: ninguno. 
Distribución: mar afuera de Baja California y Panamá (Wicksten 1989).

\section{Pleuroncodes monodon}

(H. Milne Edwards, 1837)

Material: $17 O^{\top} 10 \bigcirc$, golfo de Nicoya, 20.VII.1969 (UCR 628-01). $3 \sigma^{\top} 3$ q, mar afuera de Quepos, 7.V.1970 (UCR 636-01). 5 o 10\%, $17.6 \mathrm{~km}$ al NO de isla del Caño, 15.VII.1969

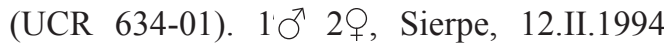
(UCR 2012-01). 20, golfo Dulce, 11.XII.1993 (UCR 2005-07). $30^{\top}$, mar adentro frente a punta Gorda,Pacífico $10^{\circ} 32^{\prime} \mathrm{N}, 86^{\circ} 24^{\prime} \mathrm{W}$, 19.VI.1970 (UCR 632-01). $20^{\top} 1$ \%, mar adentro al sur de isla Negritos, golfo de Nicoya, $9^{\circ} 40^{\prime} \mathrm{N}, 84^{\circ} 50^{\prime} \mathrm{W}, 8 . \mathrm{V} .1970$ (UCR 637-01). 1999).

Hábitat: béntico (Hendrickx y Harvey

Informes previos en CR: mar afuera de Costa Rica (Hendrickx y Harvey 1999).

Distribución: del golfo de Tehauntepec, México y de Perú a Chile (Hendrickx 1995).

Familia PORCELLANIDAE Haworth, 1825

\section{Euceramus panatelus \\ Glassell, 1938}

Material: ninguno.

Hábitat: vive al parecer debajo de la superficie de la arena, entre 9-40 m (Haig 1960).

Informes previos en Costa Rica: golfo Dulce (Haig 1960).

Distribución: de bahía Tenacatita, México hasta La Libertad, Ecuador (Haig 1960).

\section{Euceramus transversilineatus (Lockington, 1878)}

Material: $10^{\top} 1 q$, playa Mostrencal Junquillal, 13.III.1996 (UCR 2149-09).

Hábitat: en fondos de arena, conchas, conchas y arena, lodo arenoso y arena con corales, dragado entre 4-60 metros (Haig 1960).
Informes previos en CR: bahía Santa Elena, Costa Rica (Haig 1960). Frente a la desembocadura del río Barranca, golfo de Nicoya (Vargas et al. 1985).

Distribución: de Baja California hasta isla Taboga, Panamá (Abele y Kim 1989).

\section{Megalobrachium erosum (Glassell, 1936)}

Material: $1 \uparrow$ ov., Punta Morales, golfo de Nicoya, 2.VIII.1996 (UCR 2196-02).

Hábitat: dragado a profundidades de 9 a $46 \mathrm{~m}$ en fondos arenosos, arena con nulíporos, conchas y arena, rocas y arena (Haig 1960). En el litoral (Gore 1982). El espécimen aquí informado fue recolectado bajo rocas en playones de fango expuestos en marea baja en el estuario de Punta Morales, golfo de Nicoya.

Informes previos en CR: ninguno.

Distribución: de Baja California y el golfo de California a Panamá (Gore 1982).

\section{Megalobrachium festai}

(Nobili, 1901)

Material: $10^{\pi} 1 q$ ov., Cambutal, Dominical, 16.V.1995 (UCR 2032-07).

Hábitat: áreas rocosas de arrecifes, desde el litoral hasta $8 \mathrm{~m}$ (Gore 1982).

Informes previos en CR: ninguno.

Distribución: de México al Ecuador (Gore 1982).

\section{Megalobrachium garthi \\ Haig, 1957}

Material: $1 \sigma^{\top}$, Pitahaya, Guanacaste, 15.VI.1991 (UCR 1596-01).

Hábitat: debajo de rocas en el litoral, de esponjas y coral; de la orilla a $7 \mathrm{~m}$ de profundidad (Haig 1957).

Informes previos en CR: ninguno.

Distribución: de la isla Turner, golfo de California hasta puerto Utría, Colombia, incluyendo islas Tres Marías, México (Haig 1960). 
Comentario: primer informe para Costa Rica.

\section{Megalobrachium pacificum Gore \& Abele, 1973}

Material: $10^{\top}$, bahía Hachal, Penísula de Santa Elena, 7-9.XI.1992 (UCR 2058-02). 19, cabo Blanco, 10.III.1993 (UCR 189103). $10^{\pi} 1$, roca Negritos, entre Cambutal y Dominical, 17.V.1995 (UCR 2030-03). $10^{\pi}$, puerto Jiménez, 17.III.1990 (UCR 1966-01).

Hábitat: recolectado en la zona entre mareas en áreas rocosas abiertas bajo piedras sobre fondos arcillosos-lodosos (Gore y Abele 1973).

Informes previos en CR: bahía Salinas (Haig 1956, 1960); playa Blanca (Gore y Abele 1973), isla Cedro, golfo de Nicoya (Haig 1968).

Distribución: Costa Rica y Panamá (Gore 1982).

Comentario: en el Pacífico esta especie fue considerada como Megalobrachium poeyi hasta que Gore y Abele (1973) la describieron como especie nueva.

\section{Megalobrachium tuberculipes (Lockington, 1878)}

Material: ninguno.

Hábitat: se encuentra sobre esponjas incrustadas en abanicos de mar (Haig 1960), desde el litoral hasta $20 \mathrm{~m}$ (Gore 1982).

Informes previos: isla Tortuga (isla Jasper), golfo de Nicoya (Haig 1968).

Distribución: del norte de Baja California y el golfo de California al Ecuador (Gore 1982).

\section{Neopisosoma bicapillatum}

Haig, 1960

Material: $10^{\top}$, punta Sucia, entre Jacó y punta Leona, 11.V.1992 (UCR 1584-01). 1960).

Hábitat: litoral, debajo de rocas (Haig

Informes previos en CR: ninguno.

Distribución: conocido de puerto Utría, Colombia, La Libertad, Ecuador e islas Galápagos (Haig 1960).
Comentarios: primer informe para Costa Rica y se amplía la distribución al norte hasta punta Sucia, Garabito, Puntarenas.

\section{Neopisosoma dohenyi Haig, 1960}

Material: $1 \sigma^{\nearrow} 1$, , cabo Blanco, 9.II.1993 (UCR 1871-10). 1 О', cabo Blanco, 10.III.1993 (UCR 1891-08). 10', cabo Blanco, 9.II.1993 (UCR 1871-04).

Hábitat: litoral hasta $8 \mathrm{~m}$ (Haig 1960). En los intersticios de corales pocillopóridos donde el oleaje es fuerte (Werding y Haig 1982).

Informes previos en CR: ninguno.

Distribución: de la boca del golfo de California, México a la isla Gorgona, Colombia (Werding y Haig 1982).

Comentarios: primer informe para Costa Rica.

\section{Neopisosoma mexicanum}

(Streets, 1871)

Material: $3 \sigma^{\pi} 3 q$ ov., cabo Blanco, 11.II.1993 (UCR 1884-02). 19, cabo Blanco,

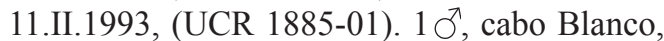
11.II.1993 (UCR 1885-02). $10^{\top} 19$ ov., Dominical, 9-10.XI.1968 (UCR 504-01).

Hábitat: litoral, debajo de rocas (Haig 1960).

Informes previos en CR: Dominical, Puntarenas (Moran y Dittel 1993).

Distribución: de Mazatlán, México hasta punta Santa Elena, Ecuador e islas Galápagos (Haig 1960).

\section{Orthochela pumila Glassell, 1936}

Material: $60^{\top} 4 \bigcirc$ (2 ov.), 3 juv., playa Sámara, Guanacaste, VII.1999 (UCR 2317-02).

Hábitat: son comensales obligatorios sobre abanicos de coral (Haig 1962), ceñidos a corales gorgónidos amarillos, donde cada espécimen empataba perfectamente con las marcas de color del gorgónido en el cual se encontra (Haig et al. 1970). 
Informes previos en CR: isla San Lucas, golfo de Nicoya (Haig 1962).

Distribución: de bahía Magdalena, en el exterior de Baja California y Mazatlán, México, a bahía de Caraquez, Ecuador (Haig et al. 1970).

\section{Pachycheles biocellatus (Lockington, 1878)}

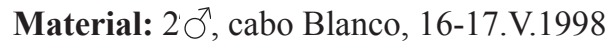

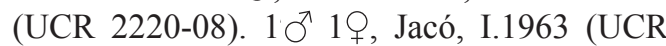
140-01). $10^{\top}$, entre Cambutal y Dominical, 16.V.1995 (UCR 2032-06). 43 O` $^{\top} 63$ (11 ov.), isla del Caño, 13.III,11.IV.1970 (UCR 363-01). $50^{\lambda} 5$ O ov., isla del Caño, 11.IV.1970 (UCR 594-01). $2 \sigma^{\nearrow} 1$ \%, isla del Caño, 26.IV.1997 (UCR 2190-02). $10^{\pi} 1$ \&, 2 juv., isla del Caño, 14.XI.1997 (UCR 2213-10). 19 ov., isla del Coco, 2.IV.1992 (UCR 1658-01). 19, isla del Coco, 7-9.IV.1992 (UCR 1656-01). $7 \sigma^{\nwarrow} 5$, isla del Coco, 8.II.1993 (UCR 1933-02).

Hábitat: del litoral hasta $26 \mathrm{~m}$ (Gore 1982), asociados a corales y también bajo rocas (Haig 1960, Werding y Haig 1982).

Informes previos en CR: bahía Santa Elena; islas Viradores, Bahía Culebra (Haig 1960).

Distribución: del golfo de California a isla La Plata, Ecuador (Gore 1982); islas Revillagigedo, Clipperton, Malpelo, Gorgona y Galápagos (Werding y Haig 1982).

Comentario: se amplía distribución a isla del Coco.

\section{Pachycheles calculosus Haig, 1960}

Material: $10^{\top} 1$ ov., cabo Blanco, 9.II.1993 (UCR 1871-05). $3 \sigma^{\top} 2$ \%, cabo Blanco, 9.III.1993 (UCR 1873-07). $10^{\top}$, cabo Blanco, 10.III.1993 (UCR 1891-02). $10^{\top} 1$, cabo Blanco, 10.III.1993 (UCR 1891-04).

Hábitat: en coral y áreas rocosas en arrecifes (Haig 1960), desde el litoral hasta $8 \mathrm{~m}$ (Gore 1982).

Informes previos en CR: bahía Salinas (Haig 1960), golfo de Nicoya (Gore 1982).
Distribución: del golfo de California hasta La Libertad, Ecuador (Gore 1982).

$$
\begin{gathered}
\text { Pachycheles chacei } \\
\text { Haig, } 1956
\end{gathered}
$$

Material: 1q, cabo Blanco, 9.II.1993 (UCR 1871-06). 19, cabo Blanco, 10.III.1993 (UCR 1891-05).

Hábitat: recolectado de coral (Haig 1960), desde el litoral hasta $8 \mathrm{~m}$ (Gore 1982).

Informes previos en CR: islas Tortuga, golfo de Nicoya y bahía Uvita (Haig, 1968).

Distribución: en el Pacífico, de Guatemala a Ecuador y en el Caribe, de Panamá a Colombia (Gore 1982).

\section{Pachycheles panamensis Faxon, 1893}

Material: $2 \sigma^{\Uparrow} 1 \%$, punta Morales, golfo de Nicoya, 2.VIII.1996 (UCR 2196-03).

Hábitat: la zona entre mareas, bajo rocas y entre los intersticios de coral (Haig 1960). Del litoral hasta los $8 \mathrm{~m}$ (Gore 1982). Los especímenes aquí informados se encontraron bajo rocas en playones lodosos expuestos durante marea baja en el estuario de punta Morales, golfo de Nicoya.

Informes previos en CR: Puntarenas (Haig 1962), bahía Coronado (Haig 1968).

Distribución: de Baja California y el golfo de California hasta Ecuador y mar adentro de la isla Isabel (Gore 1982).

\section{Pachycheles spinidactylus Haig, 1957}

Material: $1 \sigma^{\lambda}$, punta Sabana, Conchal, 4.V.1985 (UCR 1533-02). 2 o ov., playa Pitahaya, 15.IV.1991 (UCR 1444-01). $10^{\top}$, playa Pitahaya,

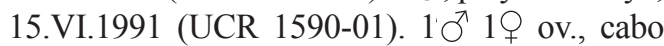
Blanco, 9.II.1993 (UCR 1871-09).

Hábitat: litoral, debajo de rocas y de esponjas, de la orilla a 8 m (Haig 1956).

Informes previos en CR: al sur de islas Viradores, bahía Culebra (Haig 1960); Puntarenas, golfo de Nicoya (Gore 1982). 
Distribución: de Baja California y golfo de California hasta Colombia (Gore 1982).

\section{Pachycheles subsetosus Haig, 1960}

Material: ninguno.

Hábitat: de 10 a 14 m (Haig 1960).

Informes previos en CR: Puntarenas, Costa Rica (Haig 1960).

Distribución: de el Salvador a Puntarenas, Costa Rica (Moran 1984).

\section{Pachycheles velerae Haig, 1960}

Material: $4 \sigma^{\top} 5$ ( 1 ov. $)$, isla del Coco,

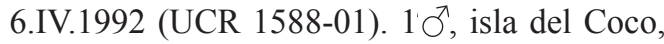
6.IV.1992 (UCR 1726-01).

Hábitat: sustrato rocoso, de $36-86 \mathrm{~m}$ (Harvey 1998). Los organismos mencionados en este trabajo fueron dragados de $121 \mathrm{~m}$ utilizando una "tangle net", recolectados por M. Montoya.

Informes previos en CR: isla del Coco (Harvey 1998).

Distribución: mar afuera de la isla Bindlae, Galápagos (Haig 1960) e isla del Coco (Harvey 1998).

\section{Pachycheles vicarius \\ Nobili, 1901}

Material: $1 q$ ov., cabo Blanco, 9.III.1993 (UCR 1876-01). 10', cabo Blanco, 9.III.1993 (UCR 1873-05). 5 (4 ov.), 2 juv., entre Cambutal y Dominical, 16.V.1995 (UCR 203205). 4 ( (3 ov.), roca Negritos, entre Cambutal y Dominical, 17.V.1995 (UCR 2030-05). 2 ( 1 ov.), isla del Caño, s.f. (UCR 1213-02). $60^{\top} 5$ O (4 ov.), isla del Caño, 25.II.1970 (UCR 408-

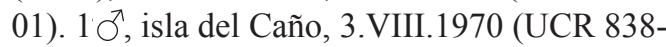
05). $480^{7} 869$ (46 ov.), 22 juv., isla del Caño, 11.IV.1970 (UCR 362-01). $10^{\top 7} 2$ ( 1 ov.), isla del Caño, 14.XI.1997 (UCR 2213-12).

Hábitat: la zona entre mareas, bajo rocas, común entre corales hasta 8 m (Haig 1960).
Informes previos en CR: bahía Santa Elena y al sur de islas Viradores, bahía Culebra (Haig 1960).

Distribución: de Acajutla, El Salvador a bahía Santa Elena, Ecuador (Gore y Abele 1976); isla Gorgona (Werding y Haig 1982).

\section{Petrolisthes agassizii \\ Faxon, 1893}

Material: $3 \bigcirc$ ( 2 ov.), playa Junquillal, Guanacaste, 20.II.1995 (UCR 2014-04). $1 O^{\top} 1$, , islas Murciélago, 5.V.1999 (UCR 2292-06). $10^{\top} 2$ \%, Brazilito, Guanacaste, 18.V.1980 (UCR 1275-01). 1q, Ostional, 1980 (UCR 837-02). $20^{\top} 1$ \% ov., cabo Blanco, 27.X.1992 (UCR 1779-01). 1\%, cabo Blanco, 10.III.1993 (UCR 1891-06). 10 1 \%, cabo Blanco, 10.III.1993 (UCR 1875-06). $20^{\Uparrow} 1$ \%, roca Negritos, entre Cambutal y Dominical, 17.V.1995 (UCR 2139-01).

Hábitat: bajo rocas durante la marea baja (Haig 1960), desde el litoral hasta $10 \mathrm{~m}$ (Gore 1982). En intersticios de coral Pocillopora (Werding y Haig 1982).

Informes previos en CR: bahía Santa Elena y playa Blanca (Haig 1968); playas del Coco y Puntarenas (Gore 1982).

Distribución: golfo de California hasta Colombia (Gore 1982) e isla Gorgona (Werding y Haig 1982).

\section{Petrolisthes armatus \\ (Gibbes, 1850)}

Material: $4 O^{\top} 8 Q$ ( 1 ov.), 2 juv., Junquillal,

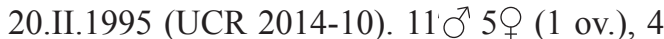
juv., estero Jicaral, 1995 (UCR 2143-01). $3 \sigma^{\top}$ 3 ov., golfo de Nicoya, 16.XII.1993 (UCR 2010-07). $4 O^{\top} 3$ \%, punta Morales, 2.VIII.1996 (UCR 2231-01). $90^{\top} 4$ 4 (3 ov.), 1 juv., punta Morales, 11.VIII.1983 (UCR 1053-02). $120^{\text {Ј }}$ $12 \bigcirc$ ov., punta Morales, 8.X.1989 (UCR 138006). $4 \bigcirc$ (1 ov.), punta Morales, 13.VIII.1992

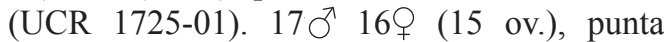
Morales, 14.VII.1992 (UCR 1751-01). 1Q,

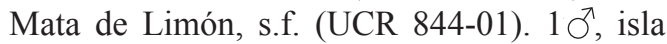


del Caño, 11.IV.1970 (UCR 358-01). 2 juv., isla del Caño, 3.VIII.1970 (UCR 1091-01). 3 o $^{\top} 2$, playa Amapola, Península de Osa,

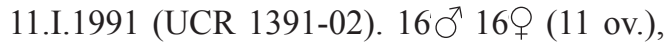
puerto Jiménez, 17.II.1990 (UCR 1817-01). 28 O $^{\top} 15$ ( $(5$ ov.), puerto Jiménez, 31.I.1997 (UCR 2194-02). $30^{\nearrow} 3$ O ov., isla Pelícano, Golfito, 18.VII.1973 (UCR 839-01).

Hábitat: tiene gran variedad de hábitats, desde el litoral hasta $30 \mathrm{~m}$ (Haig 1960).

Informes previos en CR: bahía Salinas y Puntarenas (Haig 1960), bahías Santa Elena y Culebra, isla Tortuga y bahía Coronado (Haig 1968).

Distribución: en el Pacífico del golfo de California a Perú; en el Atlántico desde África Occidental hasta Florida y en el Caribe hasta Brasil (Haig 1960).

\section{Petrolisthes artifrons Haig, 1960}

Material: ninguno.

Hábitat: bajo rocas, desde el litoral a $20 \mathrm{~m}$ (Haig 1960). En intersticios de coral (Werding y Haig 1982)

Informes previos en CR: bahía Santa Elena (Haig 1960).

Distribución: de Costa Rica a Ecuador (Gore 1982).

\section{Petrolisthes cocoensis (Haig, 1960)}

Material: ninguno.

Hábitat: en la zona entre mareas bajo rocas (Haig 1960).

Informes previos en CR: bahías Wafer y Chatham, isla del Coco (Haig 1960).

Distribución: isla del Coco (Haig 1960).

Comentarios: especie endémica de la isla del Coco.

\section{Petrolisthes edwardsii}

(Saussure, 1853)

Material: $1 O^{\pi}$, Junquillal, 20.II.1995 (UCR 2014-12). 1 ov., islas Murciélago,
7.XII.1969 (UCR 268-01). $10^{\text {T } 2} 2$ (1 ov.), isla Viradores, bahía Culebra, 9.VII.1995 (UCR 2041-01). 19 ov., playas del Coco, 13.IV.1965 (UCR 341-01). 1 ov., punta Sabana, Conchal, 5.V.1984 (UCR 1926-01). $2 O^{\pi} 19$, playa Sámara, 16.I.1984 (UCR 2216-02). 9 đ $^{\top}$ 8, 5 juv., cabo Blanco 16-17.V.1998 (UCR 2220-

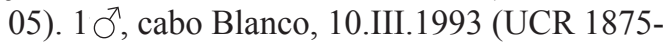
01). 1 Q ov., cabo Blanco, 18.IV.1995 (UCR 2039-01). $4 \bigcirc^{\nearrow} 5$ ( 3 ov.), Mata de Limón, 12.VIII.1967 (UCR 137-01). 19 ov., punta Leona, 1984 (UCR 1082-02). 2 o $^{\top} 2$ ( (1 ov.), isla del Caño, 1970 (UCR 447-01). 1ठ઼, isla del Caño, 7.IV.1970 (UCR 484-01). 1 juv., isla del Caño, 3.VIII.1970 (UCR 838-01). 2 O’ $^{\top}$, isla del Caño, 23.I.1984 (UCR 1009-01). 1 ov., playa Amapola, península de Osa, 11.I.1990 (UCR 1479-01). $2 \sigma^{\top} 1 \%$, playa Amapola, península de Osa, 11.I.1990 (UCR 1457-01).

Hábitat: zona entre mareas, bajo rocas y colonias de coral, hasta $40 \mathrm{~m}$ (Haig 1960).

Informes previos en CR: bahía Santa Elena, playa Blanca, y bahías Chatham y Wafer, isla del Coco (Haig 1960); bahías Santa Elena y Culebra e isla Tortuga, golfo de Nicoya (Haig 1968); golfo de Nicoya, Puntarenas (Gore 1982).

Distribución: Baja California y el golfo de California hasta Ecuador (Gore 1982), islas Revillagigedo y Galápagos (Werding y Haig 1982).

\section{Petrolisthes galapagensis \\ Haig, 1960}

Material: ninguno.

Hábitat: bajo rocas en la zona entre mareas hasta $5 \mathrm{~m}$ (Haig 1960).

Informes previos en CR: isla Tortuga, golfo de Nicoya (Haig 1960).

Distribución: México, Costa Rica e islas Galápagos (Gore 1982).

\section{Petrolisthes glasselli Haig, 1957a}

Material: $10^{\top}$, punta Islotes, Puntarenas,

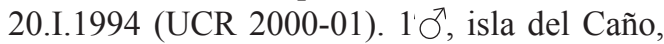




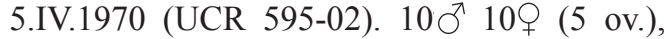
isla del Caño, 13.III.1970 (UCR 356-01). $510^{7}$ 49 (16 ov.), isla del Caño, 12.IV.1970 (UCR 611-01). 47 O $^{\top} 53 \bigcirc$ (22 ov.), 8 juv., Sierpe, 24.IX.1980 (UCR 1345-01). 1 9 , isla del Coco,

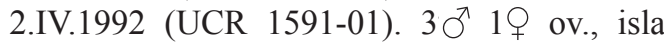
Pájaros, isla del Coco, 3.IV.1992 (UCR 160501). 6 (3 ov.), roca Sucia, Isla del Coco, 1-9.IV.1992 (UCR 1580-01). 19 ov., isla del Coco, 4.IV.1992 (UCR 1593-02). 2 o ov., isla del Coco, 5.IV.1992 (UCR 1616-01). $10^{\text {T } 2} 2$ ? (1 ov.), isla del Coco, 1-9.IV.1992 (UCR 161501). 19, isla del Coco, 3.IV.1992 (UCR 165701). $10^{\nearrow} 1$, isla del Coco, 29.III.1989 (UCR 1581-01).

Hábitat: asociado usualmente a corales como Pocillopora y Pavona, del litoral a $8 \mathrm{~m}$ (Haig 1960).

Informes previos en CR: bahía Santa Elena y cercano al sur de islas Viradores, puerto Culebra (Haig 1960); bahías Santa Elena, Culebra y Coronado (Haig 1968).

Distribución: del golfo de California hasta las islas Gorgona y Malpelo, Colombia, incluyendo islas Tres Marías, Isabel Revillagigedo, Galápagos y Clipperton (Gore y Abele 1976).

Comentario: se amplía distribución a isla del Coco.

\section{Petrolisthes haigae \\ Chace, 1962}

Material: $2 \sigma^{\pi} 2 Q$ (1 ov.), Junquillal, 20.II.1995 (UCR 2014-05). 1 ov., 6 juv., península Santa Elena, 14.IX.1970 (UCR 845-01). 1 q ov., península de Santa Elena, 9.I.1994 (UCR 1929-03). $10^{\top}$, playa Sámara,

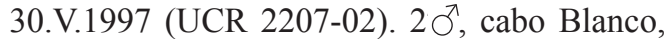
16-17.V.1998 (UCR 2220-07). 19, Jacó, I.1963 (UCR 139-01). 19 ov., Cambutal, Dominical, 16.V.1995 (UCR 2032-09). $60^{\top} 7$ (6 ov.), Cambutal, Dominical, 16.V.1995 (UCR 203209). $490^{\text {T }} 82$ ( $(52$ ov.), 3 juv., isla del Caño, 25.II.1970 (UCR 610-01). $40^{\top} 5$ 5 (3 ov.), isla

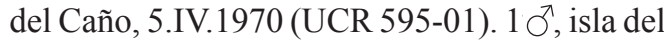
Caño, 11.IV.1970 (UCR 357-01). 104 ठ 100ㅇ (35 ov.), 42 juv., isla del Caño, 11.IV.1970 (UCR 360-01). $310^{\top} 40 \bigcirc$ (25 ov.), 3 juv., isla

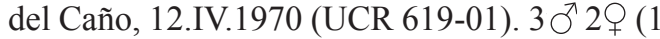
ov.), isla del Caño, 3.VIII.1970 (UCR 838-03). 1 o ov., isla del Coco, 4.IV.1992 (UCR 159301). 1 ठ઼, isla del Coco, 1-9.IV.1992 (UCR

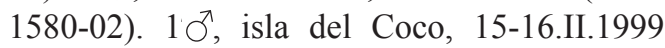
(UCR 2259-11).

Hábitat: asociado a coral (Haig 1960), del litoral hasta los $12 \mathrm{~m}$, excepcionalmente se encuentra a $44 \mathrm{~m}$ (Gore 1982).

Informes previos en CR: bahías Santa Elena y Culebra, isla Tortuga y bahía Coronado (Haig 1968); bahía Culebra y golfo de Nicoya (Gore 1982).

Distribución: del golfo de California al Ecuador y las islas Malpelo, Islabel, Tres Marías, Revillagigedo, Galápagos y Clipperton (Gore 1982).

Comentario: se amplía distribución a isla del Coco.

\section{Petrolisthes hians \\ Nobili, 1901}

Material: 19 ov., cabo Blanco, 9.11.1993 (UCR 1871-07). 1 $0^{\top}$, punta Sucia, entre Jaco y punta Leona, 5.IV.1992 (UCR 1584-03). $10^{\nearrow}$ 1 ov., Cambutal, Dominical, 16.V.1995 (UCR 2030-13).

Hábitat: asociado a colonias de coral (Haig 1960), desde el litoral hasta los $34 \mathrm{~m}$ (Gore 1982).

Informes previos en CR: bahía Santa Elena y cerca de islas Viradores (Haig 1960), bahía Culebra, isla Tortuga, golfo Nicoya y bahía Coronado (Haig 1968), golfo de Nicoya (Gore 1982).

Distribución: de Baja California al Ecuador, incluyendo las islas Isabel, Tres Marías y Revillagigedo (Gore 1982).

\section{Petrolisthes holotrichus Nobili, 1901}

Material: 1 ð’, bahía Hachal, 7-9.XI.1995 (UCR 2058-01). 2 ( $(1$ ov.), 1 juv., playa Sámara, 30.V.1997 (UCR 2207-06). $10^{\top} 1$ 1 ov., cabo Blanco, 10.III.1993 (UCR 1891-01). $2 \sigma^{\top} 1$ ov., Dominical, 9-10.XI.1968 (UCR 
189-01). $2 \sigma^{\top} 4 q$ (2 ov.), Cambutal, Dominical,

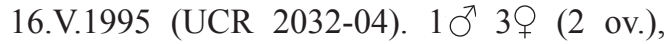
roca Negritos, entre Cambutal y Dominical, 17.V.1995 (UCR 2030-04). 1q ov., isla del Caño, 1.III.1970 (UCR 1542-05). $1 \sigma^{\top}$, isla del Caño, 26.V.1989 (UCR 1914-04). $40^{\top} 4$ ㅇ (2 ov.), isla del Caño, 25.IV.1997 (UCR 2189-06)

Hábitat: en el litoral, bajo rocas (Haig 1960).

Informes previos en CR: bahía Salinas, bahía Santa Elena y golfo Dulce (Haig 1960), bahía Ballena (Haig 1968); playas del Coco (Gore 1982).

Distribución: de El Salvador hasta Ecuador (Gore 1982).

\section{Petrolisthes lewisi Glassell, 1936}

Material: $1 O^{\top} 1 Q$ ov., playa Blanca, Parque Nacional Santa Rosa, 16.II.1995 (UCR 202004). 1 o ov., Conchal, Guanacaste, 11.II.1973 (UCR 1672-01). $10^{\top}$, cabo Blanco, 10.III.1993 (UCR 1891-06). 1 ठ', cabo Blanco, 9.III.1993 (UCR 2109-03). 19, Dominical, 9-10.XI.1968 (UCR 188-01). $30^{\top} 6$ ㅇ (4 ov.), roca Negritos, entre Cambutal y Dominical, 1995 (UCR 2030-02). $10^{\pi} 2$ ov., Cambutal, Dominical, 16.V.1995 (UCR 2032-08). 14 đ’ 13 $^{\top} 4$ juv., isla del Caño, 3.IV.1970 (UCR 359-01). 3 Q (1 ov.), isla del Caño, 25.II.1970 (UCR 610-02). 1 ơ $^{7}$ Q (2 ov.), isla del Caño, 13-14.XI.1997 (UCR 2213-16).

Hábitat: bajo las rocas en la zona entre mareas (Haig 1960).

Informes previos en CR: bahía Salinas y bahía Santa Elena (Haig 1960).

Distribución: de Nicaragua a Ecuador (Gore 1982).

Comentarios: el holotipo es de bahía Santa Elena (Haig 1960).

\section{Petrolisthes nobilii Haig, 1960}

Material: $3 \sigma^{\top} 5 \bigcirc(1$ ov.), playa Blanca, Parque Nacional Santa Rosa, 16.II.1995 (UCR
2020-04). $10^{\top} 4$ ov., cabo Blanco, s.f. (UCR 1877-03). $10^{\top}$, cabo Blanco, 9.II.1993 (UCR 1874-01). 1q ov., cabo Blanco, 10.III.1993 (UCR 1892-02). $1 \sigma^{\top}$, roca Negritos, entre Cambutal y Dominical, 17.V.1995 (UCR 2030-01). $10^{\text {T }} 19$ ov., punta Catedral, Manuel Antonio, 12.X.1980 (UCR 1660-02). $10^{7}$ $2 q$ ov., isla del Caño, 25.IV.1997 (UCR 218909). 19 ov., isla del Caño, 3.VIII.1970 (UCR 838-02). $10^{\top}$, isla del Caño, 11.II.1970 (UCR 523-01). 4 $0^{\top} 1$, Mogos, península de Osa, 18.XII.1996 (UCR 1465-01).

Hábitat: zona entre mareas, bajo rocas (Haig 1960).

Informes previos en CR: bahía Santa Elena (Haig 1960), bahía Ballena, golfo de Nicoya (Haig 1968).

Distribución: del golfo de California a Ecuador e isla Isabel (Lemaitre y AlvarezLeón 1992).

\section{Petrolisthes ortmanni Nobili, 1901}

Material: $2 \sigma^{\top} 5 q$, Cambutal, Dominical, 16.V.1995 (UCR 2032-10). 10', San Miguel, cabo Blanco, 9.III.1993 (UCR 2109-03).

Hábitat: zona litoral, bajo rocas y en coral (Haig 1960), de 0 a 7 m (Gore 1982). Comensal con Aniculus elegans (este trabajo).

Informes previos en CR: bahía Santa Elena, playa Blanca, cerca del sur de islas Viradores y bahía Chatham, isla del Coco (Haig 1960); bahía Santa Elena, isla Tortuga y bahía Coronado (Haig 1968); golfo de Nicoya (Gore 1982).

Distribución: del golfo de California a Perú e islas Tres Marías y Coco (Gore 1982).

\section{Petrolisthes platymerus Haig, 1960}

Material: $10^{\top} 49$ (1 ov.), playa Blanca, Parque Nacional Santa Rosa, 16.II.1995 (UCR 2020-05). $10^{\top}$, Conchal, 5.I.1973 (UCR 125704). $10^{\top}$, punta Sabana, Conchal, 4.V.1985 (UCR 1533-01). 
Hábitat: litoral, bajo rocas (Haig 1960).

Informes previos en CR: bahía Santa Elena (Haig 1960), bahía Ballena, golfo de Nicoya (Haig 1968).

Distribución: Costa Rica y Panamá (Gore 1982).

Comentarios: el holotipo es de bahía Santa Elena (Haig 1960).

\section{Petrolisthes polymitus \\ Glassell, 1937}

Material: $10^{\top} 1 \%$, isla del Caño, IIIIV.1970 (UCR 360-02). $60^{7} 9$ 9 (3 ov.), isla del Caño, 11.IV.1970 (UCR 361-01). 19 ov., isla del Caño, 26.IV.1998 (UCR 2229-03).

Hábitat: litoral, bajo rocas, en coral, en esponjas hasta los $8 \mathrm{~m}$ (Haig 1960).

Informes previos en CR: playa Blanca (Haig 1960), islas Tortuga y bahía de Coronado (Haig 1968), playas del Coco y golfo de Nicoya (Gore 1982).

Distribución: del golfo de California a Ecuador, incluyendo las islas Tres Marías y Galápagos (Gore 1982).

\section{Petrolisthes robsonae Glassell, 1945}

Material: 1Q, Jicaral, 28.II.1985 (UCR 1245-06). 2 , Jicaral, 28.II.1985 (UCR 124709). $2 O^{\top}$, Jicaral, 28.II.1985 (UCR 1244-06). 1 ov., Jicaral, 28.II.1985 (UCR 1248-05). 20 3 ㅇ, Jicaral, 30.VIII.1985 (UCR 1222-09). $10^{\top}$, Jicaral, 28.XI.1985 (UCR 1243-05). $20^{\top} 3$ \% , 1 juv., golfo de Nicoya, 28.II.1992 (UCR 166101). $40^{\top}$, Boca Guarumal, manglar de Térraba-

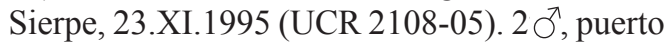
Jiménez, 31.I.1997 (UCR 2194-03).

Hábitat: capaz de soportar grandes cambios en la salinidad (Haig 1960, 1968, Gore 1982). Asociado a lodo y hojas de mangle del litoral hasta $6 \mathrm{~m}$ (Gore 1982).

Informes previos en CR: ninguno.

Distribución: México, El Salvador, Panamá y Ecuador (Gore 1982).

Comentarios: primer informe para Costa Rica.

\section{Petrolisthes tonsorius}

Haig, 1960

Material: $60^{\top} 6 \bigcirc$ (4 ov.), Mogos, Península de Osa, 18.XII.1990 (UCR 1466-01). 10 7 7,8 juv., bahía Wafer, isla del Coco, 15-16.II.1999 (UCR 2259-10).

Hábitat: debajo de rocas en el litoral (Haig 1960). De la orilla a 18 m (Haig 1968).

Informes previos en CR: bahía Santa Elena, mar afuera al sur de islas Viradores, bahía Culebra; bahía Waffer, isla del Coco (Haig 1960); isla Tortuga, golfo de Nicoya (Haig 1968).

Distribución: del golfo de California a punta Santa Elena, Ecuador (Haig 1968).

\section{Petrolisthes tridentatus Stimpson, 1858}

Material: 1 ठ', Ostional, 1980 (UCR 837 01). $10^{\Uparrow} 3 \bigcirc$ ov., s.f. (UCR 990-01). 20 , punta Catedral, Manuel Antonio, 12.X.1980 (UCR 1660-01). $10^{\top} 1$, , roca Negritos, entre Cambutal y Dominical, 17.V.1995 (UCR 2030-06).

Hábitat: litoral, bajo rocas (Haig 1960, Gore 1982).

Informes previos en CR: bahía Salinas y bahía Santa Elena (Haig 1960); bahía Santa Elena, isla Cedros, isla Tortuga y bahía Ballena (Haig 1968).

Distribución: en el Pacífico de Nicaragua a Ecuador y en el Atlántico de Bahamas a Venezuela (Gore 1982).

\section{Petrolisthes zacae Haig, 1968}

Material: $3 \sigma^{\top} 3 q$, manglar de Rincón de Osa, 17.VII.1990 (UCR-2232-01). $20^{\top} 2$, manglar de Rincón de Osa, 20.VIII.1990 (UCR 2227-01). $30^{\top} 3$ \% (2 ov.), Manglar Esquinas, Golfo Dulce, s.f. (UCR 1424-01).

Hábitat: vive en madrigueras y depresiones hechas en los bancos de lodos altos $\mathrm{y}$ expuestos que se forman en los manglares (Gore y Abele 1976). 
Informes previos en CR: bahía Ballena, golfo de Nicoya (Haig 1968).

Distribución: Costa Rica y Panamá (Gore y Abele 1976).

Comentarios: el holotipo procede de bahía Ballena, golfo de Nicoya (Haig 1968).

\section{Pisidia magdalenensis \\ (Glassell, 1936)}

Material: $1 \overbrace{}^{\top}$, Junquillal, 20.II.1995 (UCR 2014-03). 1 ov., playa Las Peladas, Nosara, 23.VII.1995 (UCR 2170-03). $60^{\top} 69$ ov., golfo de Nicoya, 23.IV.1981 (UCR 836-02).

Hábitat: común en aguas profundas en sustratos variables, pero se puede encontrar bajo las rocas en la zona entre mareas (Haig 1960). De la zona entre mareas hasta $76 \mathrm{~m}$ (Gore y Abele 1976). En las partes muertas de colonias aisladas de Pocillopora (Werding y Haig 1982). Los especímenes recolectado en playa Las Peladas, Nosara, Guanacaste, eran comensales con Aniculus elegans y Porcellana paguriconviva en una concha de cambute Strombus galeatus.

Informes previos en CR: bahía Salinas, bahía Santa Elena, playa Blanca, bahía Culebra, mar afuera al sur de islas Viradores, playa del Coco y Puntarenas (Haig 1960). Bahía Santa Elena, bahía Culebra, isla Cedros, golfo de Nicoya, Golfito, golfo Dulce (Haig 1968).

Distribución: de Baja California a Tumbes, Perú (Gore y Abele 1976) e isla Gorgona (Werding y Haig 1982).

\section{Polyonyx nitidus Lockington, 1878}

Material: 1 đั, bahía Junquillal, 1996 (UCR 2148-02).

Hábitat: dragados de fondo cubierto con hojas de manglar a $6 \mathrm{~m}$ (Haig 1960). Viviendo en ramas de colonias de Pocillopora (Werding y Haig 1982). El espécimen aquí informado fue dragado de $33 \mathrm{~m}$.

Informes previos en CR: ninguno.
Distribución: de la parte superior del golfo de California, México, a la isla Gorgona, Colombia (Werding y Haig 1982).

Comentarios: primer informe para Costa Rica.

\section{Porcellana cancrisocialis Glassell, 1935}

Material: 19, golfo de Nicoya, 23.IV.1981

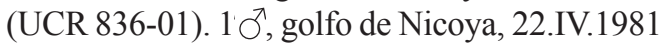
(UCR 2053-01). 19 ov., golfo de Nicoya, 15.II.1994 (UCR 2028-03). 1 ठ’, Parque Nacional Manuel Antonio, 21.XI.1996 (UCR 2182-01). 2 O’, isla del Coco, IV.1975 (UCR 1719-01).

Hábitat: observado como comensal con cangrejos ermitaños de los géneros Dardanus y Petrochirus, y libres sobre fondos de diferentes composiciones (Haig 1960). De la orilla a 108 m (Gore y Abele 1976).

Informes previos en CR: bahía Salinas, bahía Santa Elena, bahía Culebra y playas del Coco (Haig 1960); bahías Santa Elena y Culebra (Haig 1968).

Distribución: de Baja California a Tumbes, Perú (Gore y Abele 1976).

Comentarios: se amplía distribución a la isla del Coco.

\section{Porcellana paguriconviva Glassell, 1936}

Material: $2 \sigma^{\top} 1 q$ ov., isla del Coco, IV.1975 (UCR 1686-01).

Hábitat: comensal con el cangrejo ermitaño Petrochirus californiensis, de la orilla a 100 m (Gore y Abele 1976).

Informes previos en CR: bahía Salinas, bahía Santa Elena, bahía Culebra y playas del Coco (Haig 1960); bahías Santa Elena y Culebra (Haig 1968).

Distribución: de Baja California a islas Taboga y Taboguilla, bahía de Panamá (Gore y Abele 1976).

Comentarios: se amplía distribución a la isla del Coco. 


\section{Ulloaia perpusilla \\ Glassell, 1938a}

Material: ninguno.

Hábitat: entre corales gorgónidos, esponjas y briozoarios (Haig 1960). Entre cálices del coral Pocillopora damicornis, de la orilla hasta 16 m (Gore y Abele 1976).

Informes previos en CR: Puntarenas, Costa Rica (Haig 1960).

Distribución: del golfo de California a las islas Perlas, Panamá (Gore y Abele 1976).

Familia ALBUNEIDAE Stimpson, 1858

Albunea lucasia

(de Saussure, 1853)

Material: $10^{\top}$, Conchal, 11.V.1975 (UCR 1502-01).

Hábitat: de la zona intermareal a $45 \mathrm{~m}$, se encuentra en o cerca de las playas (Hendrickx 1995).

Informes previos en CR: ninguno.

Distribución: de cabo San Lucas y punta Lobos, Baja California, México, al Perú (Lemaitre y Alvarez-León 1992).

Comentarios: primer informe para Costa Rica.

\section{Lepidopa deamae}

Benedict, 1903

Material: 1q, Mata deLimón, 12.VIII.1967 (UCR 76-01).

Hábitat: vive en la zona de rompientes de playas arenosas, enterrado en la arena o nadando en el agua (Hendrickx 1995).

Informes previos en CR: Puntarenas (Gordon 1938); Mata de Limón (Efford 1971).

Distribución: del golfo de Tehuantepec hasta Colombia (Lemaitre y Alvarez-León 1992).

\section{Lepidopa mearnsi \\ Benedict, 1904}

Material: 1 , playa Naranjo, Santa Rosa, 17.II.1995 (UCR 2021-01). 1q, Ostional,
18.VII.1995 (UCR 2049-02). 19, playa Espadilla, Manuel Antonio, 9.V.1980 (UCR 1922-02).

Hábitat: debajo de arena fina en la línea inferior de marea (Haig 1980).

Informes previos en CR: Mata de Limón (Efford 1971).

Distribución: del golfo de California a Colombia (Lemaitre y Alvarez-León 1992).

\section{Lepidopa mexicana Efford, 1971}

Material: 19, Conchal, 7.V.1976 (UCR 1064-01). 1\%, Mata de Limón, 14.VIII.1985 (UCR 1035-01).

Hábitat: fondos arenosos (Efford 1971).

Informes previos en CR: Puntarenas (Efford 1971); Puntarenas, Jacó y playa Cocal, Quepos (Dexter 1974).

Distribución: del norte de Acapulco, México a Colombia (Lemaitre y Alvarez-León 1992).

Familia HIPPIDAE Latreille, 1825

\section{Emerita rathbunae}

Schmitt, 1935

Material: 12ᄋ, Junquillal, 20.II.1995 (UCR 2014-15). 19, playas del Coco, I.1976 (UCR 1253-01). 29, Conchal, 9-11.V.1975 (UCR 1270-01). 4ㅇ (2 ov.), Conchal, 9-12.V.1974 (UCR 1265-01). $120^{7}$ 59 (1 ov.), Conchal, 7.V.1976 (UCR 98801). 19, Conchal, 1.V.1973 (UCR 1598-01). 19, Conchal, V.1973 (UCR 1508-01). $10^{\top} 1$ q, Conchal, s.f. (UCR 1273-02). 2 , Ostional, 18.VII.1995 (UCR 2049-03). 3 (1 ov.), Puntarenas, 30.X.1966 (UCR 848-01). 1 ठ $^{\text {厂 }}$ $30 \bigcirc$ (3 ov.), Puntarenas, 10.IX.1970 (UCR 881-01). 19, Mata de Limón, Puntarenas, 12.VIII.1967 (UCR 75-01). 19 ov., Quepos, 24.VIII.1981 (UCR 1640-01). 1 juv., Manuel Antonio, 3.II.1995 (UCR 2018-02). 100 juv., puerto Escondido, Manuel Antonio, 4.II.1995 (UCR 2015-03). 1\%, playa Espadilla, Manuel Antonio, 9.V.1980 (UCR 1922-01). 
Hábitat: en la zona de rompientes en playas arenosas, se entierra en la arena al retirarse la ola (Hendrickx 1995).

Informes previos en CR: playas del Coco, Tamarindo, Puntarenas, boca del río Barranca, Jacó; playas Cocal y Espadilla, Quepos (Dexter 1974). Playas del Coco, Puntarenas y Mata de Limón (Efford 1976).

Distribución: de bahía San Francisco, golfo de California hasta Iquique, Chile e islas Galápagos (Haig 1980).

\section{Hippa pacifica}

(Dana, 1852)

Material: $2 \sigma^{\top} 2 Q$, bahía Caldera, 5.VII.1965 (UCR 882-01). 1ㅇ ov., isla del Caño, 3.III.1970 (UCR 432-01). $20^{\top} 4$, isla del Caño, 8.III.1970 (UCR 843-01). 20 6 ㅇ (3 ov.), 1 juv., bahía Waffer, isla del Coco, 27.III.1978 (UCR 1673-14).

Hábitat: zona intermareal de las playas y ocasionalmente a profundidades moderadas (Haig 1980).

Informes previos en CR: Puntarenas (Boone 1930), isla del Coco (Efford 1973).

Distribución: de Sonora, México a Panamá, incluyendo las siguientes islas: Revillagigedo, Clipperton, Coco y Galápagos (Haig 1980).

\section{Hippa strigillata (Stimpson, 1860)}

Material: $10^{x} 2 q$, Conchal, s.f. (UCR 1273-01). $40^{\top} 2$ (1 ov.), isla del Caño, 3.III.1970 (UCR 432-01).

Hábitat: en arena en la zona entre mareas (Effort 1973).

Informes previos en CR: Conchal, Guanacaste e isla del Caño (Moran y Dittel 1993).

Distribución: de Frailes y cabo San Lucas, golfo de California, México, hasta la isla del Caño, Costa Rica (Moran y Dittel 1993).
Super Familia Paguroidea Latreille, 1802

Familia COENOBITIDAE Dana, 1815

\section{Coenobita compressus}

H. Milne Edwards, 1837

Material: $1 \overbrace{}^{\top}$, playa Jobo, La Cruz, 7.VI.1992 (UCR 1746-01). $20^{\top} 2$ \%, playa Jobo, La Cruz, 6.VI.1993 (UCR 1747-01). 1 ठ $^{\top} 2$ \%, playa Jobo, La Cruz, 6.VI.1992 (UCR 174801). $5 O^{\top} 3$, isla San José, Islas Murciélago 14.IX.1969 (UCR 866-01). $10^{\top}$, playa Conchal, 1.V.1973 (UCR 1752-01). $120^{7} 12$ ㅇ (4 ov.),

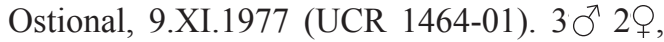
Ostional, 8.V.1977 (UCR 1420-01). 10 (7 ov.), Ostional, 16.IX.1972 (UCR 1468-01). 12 (4 ov.), Ostional, 23.IX.1972 (UCR 1470-01). $2 \sigma^{\top}$ 1q, Ostional, 23.XI.1971 (UCR 1346-01). $10^{7}$ 10Q, Ostional, 29.X.1972 (UCR 1361-01). 42 ठ 47으, Ostional, 3.I.1973 (UCR 1462-01). 5 ठ $^{7}$ 4, Ostional, 27.II.1977 (UCR 1418-01). 10@ (3 ov.), Ostional, 27.II.1977 (UCR 1471-01). 6 o $^{\top}$ 39, Ostional, 17.III.1977 (UCR 1419-01). 8 万ొ 21 , Ostional, 19.IV.1977 (UCR 1360-01). 8 ơ 10$^{\top}$, Ostional, 3.IV.1977 (UCR 1417-01).

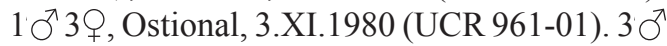
3\%, Ostional, 1980 (UCR 1031-01). $10^{\top} 2$, Ostional, 6.X.1991 (UCR 1744-01). $80^{\pi} 3$, cabo Blanco, 18.XII.1992 (UCR 1910-01). $20^{\top}$ 3, cabo Blanco, 23.XII.1992 (UCR 2132-01). $10^{\top}$, punta Morales, 30.VIII.1985 (UCR 121401). 12 O $^{\top} 16$ q, boca del río Tárcoles, 9.IV.1967

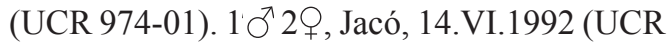
1773-01). $3 O^{\pi}$ 3ㅇ, Manuel Antonio, 3.II.1995 (UCR 2018-01). $50^{\top} 1$ \%, puerto Escondido, Manuel Antonio, 4.II.1995 (UCR 2015-02).

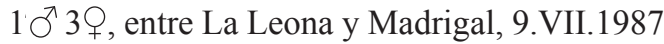
(UCR 1283-01). $10^{\top} 4 \%$, playa Amapola, península de Osa, 11.I.1990 (UCR 1480-01). $70^{\top} 6$, playa Karate, península de Osa, 21. IX. 1990 (UCR 2193-01). $20^{\top} 1$ 1 , boca de quebrada Unión, playa Karate, península de Osa. $44 O^{\top \top} 41$ ㅇ (2 ov.), isla del Caño, 1.II.1970 (UCR 1510-01). 1 $0^{\top}$, isla del Caño, 7.II.1970 (UCR 1210-01). 150 ठ 113 \%, isla del Caño, 
7.II.1970 (UCR 851-01). $20^{\top} 3$ \%, isla del

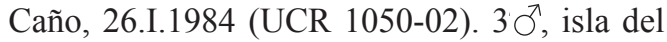
Coco, 14.VIII.1973 (UCR 621-01). $30^{\pi} 3$ q, bahía Wafer, isla del Coco, 20.II.1970 (UCR 1208-01).

Hábitat: Semiterrestre, en playas arenosas (Snyder-Conn 1980).

Informes previos en CR: Costa Rica (Pesta 1931), isla del Coco (Boone 1932), playas del Coco, isla San Lucas y Golfito (Bright 1966), isla Negritos, golfo de Nicoya y bahía Brasilito (Ball 1972).

Distribución: Del golfo de California a Perú (Snyder-Conn 1980).

Familia DIOGENIDAE Ortmann, 1892

Allodardanus rugosus

Haig y Provenzano, 1965

Material: ninguno.

Hábitat: Sustrato de arena gruesa blanca, de 73-84 m de profundidad (Haig y Provenzano 1965).

Informes previos en CR: Bahía Chatham, isla del Coco (Haig y Provenzano 1965).

Distribución: Conocido sólo en la localidad tipo (Haig y Provenzano 1965).

Comentario: especie endémica de la isla del Coco.

\section{Aniculus elegans \\ Stimpson, 1859}

Material: $10^{\lambda}$, isla San José, islas Murciélago, 5-7.XII.1969 (UCR 864-01). $10^{\top}$, playa Las Peladas, Nosara, 23.VII.1995 (UCR 2170-01). 19, cabo Blanco, 9.III.1993

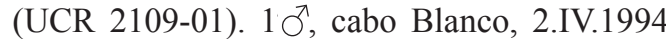
(UCR 1993-01). 19, Parque Nacional Manuel Antonio, 28.VI.1970 (UCR 1565-01). 1ㅇ, punta Ballena, isla del Caño, 24.I.1984 (UCR 1010-01). 1 ○', isla del Caño, 28.VI.1997 (UCR 2188-01). $1 \mathrm{O}^{\top}$, isla del Caño, 23.I.1984 (UCR 1044-01). 1 , , isla del Caño, 24.I.1984 (UCR

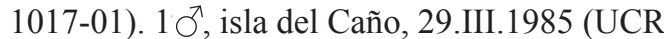
1083-01). $10^{7}$, isla del Coco, 2.IV.1992 (UCR 1670-01).
Hábitat: Sobre arena y grava, mezclas de rocas, arena y coral del género Pocillopora, a profundidades de $10 \mathrm{~m}$ (Ball y Haig 1974).

Informes previos en CR: bahía Brasilito (Ball y Haig 1974).

Distribución: de Baja California hasta Ecuador (Snyder-Conn 1980).

Comentario: Se amplía distribución a isla del Coco.

\section{Calcinus explorator \\ Boone, 1932}

Material: ninguno.

Hábitat: habita entre las rocas de la zona intermareal(Snyder-Conn 1980).

Informes previos en CR: isla del Coco (Boone 1932).

Distribución: de Baja California hasta Colombia, incluyendo las islas Tres Marías, Isabel, Galápagos, Coco, Revillagigedo y Clipperton (Snyder-Conn 1980).

\section{Calcinus obscurus Stimpson, 1859}

Material: $30^{\top}$, playa Panamá, Guanacaste, 3.XII.1980 (UCR 840-01). 14 O $^{\top} 6$ ( 2 ov.), cabo Blanco, 8.II.1993 (UCR 1883-04). 3 ơ 3 O (2 ov.), cabo Blanco, 9.II.1993 (UCR

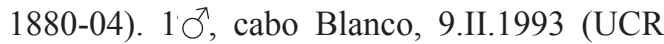
1874-03). $20^{\top}$, cabo Blanco, 9.II.1993 (UCR 1887-01). 23 ơ $9^{\text {T }}$ (2 ov.), playa Doña Ana, Puntarenas, 14.XI.1970 (UCR 865-01). 1 ㅇ ov., playa Herradura, 2.IX.1983 (UCR 196401). $2 O^{\top} 19$, puerto Escondido, Manuel Antonio, 4.II.1995 (UCR 2015-05). $20^{\circ}$ 19, isla del Caño, 3.XII.1970 (UCR 83804). $30^{\top} 3$, punta Salsipuedes, península de

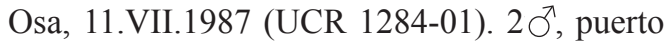
Jiménez, 17.XII.1990 (UCR 1370-06). $20^{7}$, playa Amapola, península de Osa, 11.II.1993 (UCR 1480-03). $10^{\top} 1$ \%, Mogos, península de Osa, 18.XII.1990 (UCR 1368-06).

Hábitat: muy abundante en la parte más baja de la zona intermareal (Ball y Haig 1974).

Informes previos en CR: bahía Brasilito e isla Negritos (Ball y Haig 1974). 
Distribución: de La Libertad, El Salvador a bahía Santa Elena, Ecuador (Ball y Haig 1974).

\section{Cancellus tanneri \\ Faxon, 1893}

Material: ninguno.

Hábitat: en una cavidad de una roca de coral muerto (Faxon 1895).

Informes previos en CR: isla del Coco (Faxon 1893).

Distribución: banco Gorda, Baja California Sur, golfo de California, México a islas Perlas, Panamá e islas Galápagos (Hendrickx y Harvey 1999).

\section{Clibanarius albidigitus \\ Nobili, 1901}

Material: $3 \bigcirc^{\top} 39$ ( 2 ov.), cabo Blanco, s.f. (UCR 2138-01). $2 \mathrm{O}^{\top} 4$ ㅇ (3 ov.), estero de Jicaral, 1995 (UCR 2143-03). 20 ', punta Morales, 17.VIII.1984 (UCR 1389-01). $13 \sigma^{\nearrow} 12$ (4 ov.), playa Doña Ana, Puntarenas, 10.X.1970 (UCR 869-01). 2 ठ’, Manuel Antonio, 5.II.1995

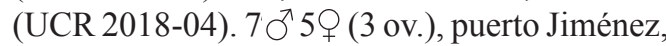
17.XII.1990 (UCR 1370-05).

Hábitat: bajo rocas en el medio de la zona intermareal (Snyder-Conn 1980).

Informes previos en CR: playa Doña Ana (Moran y Dittel 1993).

Distribución: de los Cóbanos, El Salvador a Paita, Perú (Moran 1984).

\section{Clibanarius panamensis Stimpson, 1859}

Material: 1 juv., punta El Barco, cabo Blanco, 10.III.1993 (UCR 1875-03). $30^{7}$, manglar Abangaritos, 17.I.1990 (UCR 139502). 19 , Níspero, salida al Ferry Tempisque, 16.XII.1975 (UCR 1124-02). $40^{\top} 6$ \% (2 ov.), estero Jicaral, 1995 (UCR 2143-02). $50^{7}$ 26 (10 ov.), boca estero punta Morales,

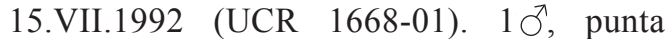
Morales, 11.VIII.1983 (UCR 1053-01). $21 ठ^{7}$ 10 ㅇ (3 ov.), punta Morales, 13.VII.1992 (UCR 1669-01). $3 \mathrm{O}^{\top}$, estero de Cocorocas, punta

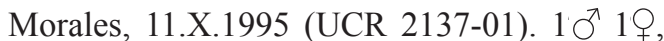
Mata de Limón, 21.VIII.1967 (UCR 871-01). $10^{\top}$, Mata de Limón, 3.XII.1980 (UCR 989-01). 3 ơ $^{\top}$ Q ov., Mata de Limón, 7.IX.1969 (UCR 2214-01). $1 O^{\nearrow} 1$ \%, bahía Caldera, VIII.1965 (UCR 853-02). $10^{\top}$, manglar del río Sierpe, 23.I.1990 (UCR 1390-02). 17 ठ 15 (11 ov.),

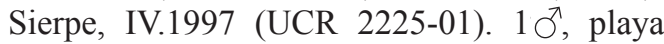
Amapola, península de Osa, 11.I.1990 (UCR 1480-02). $20^{\top} 2$ \%, 1 juv., Rincón de Osa,

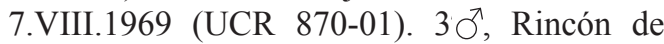
Osa, 20.VIII.1990 (UCR 1362-10). $60^{\top} 4$ ㅇ (1 ov.), Mogos, península de Osa, 18.XII.1990

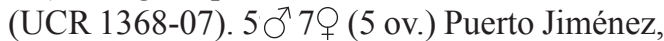
península de Osa, 17.XII.1990.

Hábitat: en agrupaciones rocosas en la parte más baja de la zona entre mareas (Ball y Haig 1974).

Informes previos en CR: punta Morales, $2 \mathrm{~km}$ al este de cerro Níspero en Ferry Tempisque, Mata de Limón, Caldera, Rincón de Osa (Moran y Dittel 1993).

Distribución: de Baja California a la bahía Cholla, Perú (Snyder-Conn 1980).

\section{Dardanus sinistripes \\ (Stimpson, 1859)}

Material: $1 \sigma^{\top} 19$, frente a Loras, golfo de Nicoya, 2.VIII.1978 (UCR 1804-01). 37 o $^{\pi} 13$ ( 3 ov.), isla Negritos, golfo de Nicoya, 19.VI.1972 (UCR 962-01). $100^{\top}$, isla Negritos, golfo de Nicoya, 19.VI.1972 (UCR 962-02). 1 o $^{\top}$, frente a Chomes, golfo de Nicoya 16.I.1998 (UCR 2212-03). $40^{\top}$ 3 ( 2 ov.), golfo de Nicoya, 7-9.III.1967

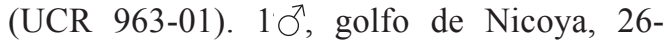
27.XII.1967 (UCR 1209-01). 19, golfo de Nicoya, 15.XII.1993 (UCR 1996-01). 5 O $^{\top} 2$, boca del río Barranca, 12.XI.1969 (UCR 883-

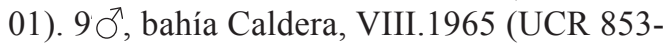
01). $10^{\pi}$, Parque Nacional Manuel Antonio, 21.XI.1996 (UCR 2182-06). 19, isla de Caño, 19.X.1984 (UCR 1066-08). 29, golfo Dulce, 12.XII.1993 (UCR 1995-05).

Hábitat: en sustratos con arena y grava, de 2-5 $\mathrm{m}$ de profundidad, pero se ha encontrado a profundidades de 110 m (Snyder-Conn 1980). 
Informes previos en CR: bahía Brasilito (Ball y Haig 1974).

Distribución: de Baja California hasta Perú (Snyder-Conn 1980).

\section{Paguristes bakeri Holmes, 1900}

Material: $3 \sigma^{\top}$, desembocadura río Tárcoles, 8-11.IX.1970 (UCR 986-02). $10^{\top}$ 2ᄋ, golfo Dulce, 10.XI.1994 (UCR 1970-01). $20^{\top}$, desembocadura de los ríos Terraba y Sierpe 13.XII.1993 (UCR 2256-01).

Hábitat: asociado con sustratos arenosos, común en aguas profundas (40 a $232 \mathrm{~m}$ ) (Snyder-Conn 1980).

Informes previos en CR: $2 \mathrm{~km}$ mar afuera de la boca del Río Tárcoles, informada como P. holmesi (Morán y Dittel 1993).

Distribución: de San Francisco, California hasta Guaymas, Perú (Snyder-Conn 1980).

Comentarios: según Hendrickx et al. (1997), P. holmesi Glassell es sinónimo menor de $P$. bakeri Holmes.

\section{Paguristes digueti Bouvier, 1893}

Material: $20^{\top}$, bahía Culebra, 19.XI.1994 (UCR 1999-02).

Hábitat: en fondos arenosos sublitorales, entre 13-60 m de profundidad (Snyder-Conn 1980).

Informes previos en CR: ninguno.

Distribución: de Baja California hasta Ecuador (Snyder-Conn 1980).

Comentarios: primer informe para Costa Rica.

\section{Paguristes fecundus} Faxon, 1893

Material: ninguno.

Hábitat: en rocas a 120 m (Faxon 1895).

Informes previos en CR: isla del Coco (Faxon 1893).

Distribución: isla del Coco (Faxon 1893)
Comentarios: la especie es referida a isla Malpelo por Lemaitre y Alvarez-León (1992), pero Faxon (1895) escribe que el material procede de la estación \#3368 comprendida entre las estaciones muestreadas mar afuera de isla del Coco. Especie endémica de la isla del Coco.

\section{Paguristes praedator} Glassell, 1937

Material: $10^{\top}$, golfo de Nicoya, 2027.XII.1967 (UCR 985-02). $20^{\top}$, golfo de Nicoya, 21.I.1987 (UCR 1277-06).

Hábitat: en fondos a profundidades de 60 m o más (Haig et al. 1970).

Informes previos en CR: golfo de Nicoya (Moran y Dittel 1993).

Distribución: Baja California y el golfo de California, México (Haig et al. 1970). Costa Rica (Moran y Dittel 1993).

\section{Petrochirus californiensis Bouvier, 1895}

Material: $10^{\top}, 5 \mathrm{~km}$ al sur de playas del

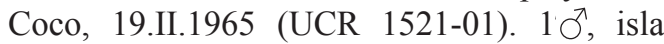
Negritos, golfo de Nicoya, 18.VI.1971 (UCR 1211-01). $10^{\top} 2$, , isla Negritos, golfo de

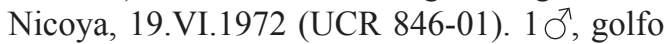
de Nicoya, 7-9.III.1967 (UCR 867-01). $10^{7}$, golfo de Nicoya, XII.1993 (UCR 1987-05).

Hábitat: fondos lodosos, raramente con mezcla de limo o arcilla, desde la zona intermareal hasta $110 \mathrm{~m}$ (Hendrickx 1995).

Informes previos en CR: bahía Brasilito e isla Cedros, golfo de Nicoya (Ball y Haig 1974).

Distribución: de Baja California hasta Ecuador (Snyder-Conn 1980).

\section{Trizopagurus magnificus \\ (Bouvier, 1898)}

Material: $1 q$ ov., islas Murciélagos,

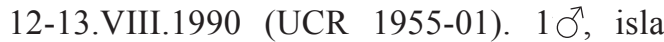
Palmitas, Guanacaste, 16.XI.1991 (UCR 158501). $30^{\pi} 2$ ( 1 ov.), cabo Blanco, 8.II.1993

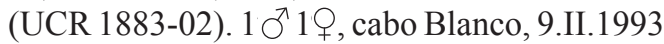


(UCR 1871-08). 10 ', cabo Blanco, 10.III.1993 (UCR 1891-09). 19, cabo Blanco, 9.II.1993

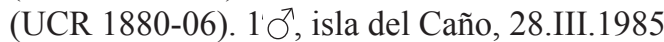
(UCR 1086-01). 10 $0^{\top}$, isla del Caño, 23.I.1984 (UCR 1044-03).

Hábitat: principalmente en afloramientos rocosos y en colonias del coral Pocillopora, desde la zona entremareas hasta los $9 \mathrm{~m}$ (Ball y Haig 1974).

Informes previos en CR: isla Negritos y bahía Brasilito (Ball y Haig 1974).

Distribución: golfo de California hasta isla La Plata, Ecuador (Ball y Haig 1974).

Familia LITHODIDAE Samouelle, 1819

Neolithodes diomedae

(Benedict, 1894)

Material: ninguno.

Hábitat: $640-2450 \mathrm{~m}$ (MacPherson 1988).

Informes previos en CR: informado por Faxon (1895) como Lithodes sp. para la isla del Coco (Boone 1926).

Distribución: golfo de California; archipiélago Chonos (Hendrickx y Harvey 1999), de Chile a Perú (MacPherson 1988).

\section{Paralomis longipes Faxon, 1893}

Material: ninguno.

Hábitat: 1000 m (Faxon 1895).

Informes previos en CR: Pacífico de Costa Rica (Moran y Dittel 1993).

Distribución: de Colombia y Perú a Iquique, Chile; isla del Coco (Haig 1974, Wicksten 1989, Moran y Dittel 1993, Hendrickx y Harvey 1999).
Familia PAGURIDAE Latreille, 1803

\section{Catapagurus diomedeae \\ Faxon, 1893}

Material: ninguno.

Hábitat: a $333 \mathrm{~m}$ de profundidad (Faxon 1895).

Informes previos en CR: isla del Coco (Hendrickx y Harvey 1999).

Distribución: de bahía Santa Inés a Banco Arena, Baja California, golfo de California, México y la bahía de Panamá; isla del Coco (Hendrickx y Harvey 1999).

\section{Enallopaguropsis janetae McLaughlin, 1982}

Material: ninguno.

Hábitat: tomado de $146 \mathrm{~m}$ de profundidad (McLaughlin 1982).

Informes previos en CR: $2.5 \mathrm{~km}$ al noroeste de punta Gissler, isla del Coco (McLaughlin 1982).

Distribución: conocido sólo de la localidad tipo, isla del Coco (McLaughlin 1982).

\section{Iridopagurus occidentalis}

(Faxon, 1893)

Material: ninguno.

Hábitat: de 22 a $121 \mathrm{~m}$, habitando conchas de Phos (Metaphos) articulatus (GarcíaGómez 1983).

Informes previos en CR: isla del Coco (Faxon 1893).

Distribución: del golfo de California a Colombia (Lemaitre y Alvarez-León 1992), isla del Coco (Faxon 1893) e islas Galápagos (García-Gómez 1983). 
Manucomplanus longimanus

(Faxon, 1893)

Material: ninguno.

Hábitat: de 37-121 $\mathrm{m}$ de profundidad (Lemaitre y McLaughlin 1996).

Informes previos en CR: isla del Coco (Faxon 1893), bahía Chatham e isla Nuez, isla del Coco (Lemaitre y McLaughlin 1996).

Distribución: del golfo de California, México hasta mar afuera frente a Panamá (Lemaitre y McLaughlin 1996).

\section{Pagurus albus}

(Benedict, 1892)

Material: $1 O^{\top} 3 Q$ ov., golfo de Nicoya,

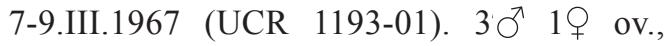
isla Negritos, golfo de Nicoya, 23.II.1978 (UCR 1805-01). $10^{\top} 4$ ㅇ ov., golfo de Nicoya, 15.II.1994 (UCR 1994-05).

Hábitat: fondos arenosos (Snyder-Conn 1980).

Informes previos en CR: Costa Rica (Boone 1930, Hendrickx y Harvey 1999).

Distribución: de puerto Peñasco hasta bahía Santa Inés, golfo de California (SnyderConn 1980); Costa Rica (Boone 1930).

Pagurus gladius

(Benedict, 1892)

Material: $4 O^{\top} 6 \bigcirc$ (5 ov.), golfo de Nicoya, 10.IV.1980 (UCR 779-09). $2 \mathrm{O}^{\top}$, golfo de Nicoya, 11.IV.1980 (UCR 781-06). 1\%, isla Negritos, golfo de Nicoya, 19.VI.1972(UCR

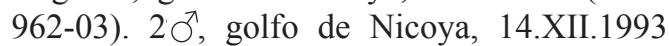
(UCR 1980-08).

Hábitat: de 36-91 m de profundidad (Snyder-Conn 1980).

Informes previos en CR: golfo de Nicoya (Maurer et al. 1984).
Distribución: del golfo de California a Ecuador (Snyder-Conn 1980).

\section{Pagurus lepidus \\ (Bouvier, 1898)}

Material: $130^{\top} 69$ (1 ov.), San Miguel, Cabo Blanco, 16-17.V.1998 (UCR 2220-12).

Hábitat: común en la parte más baja de la zona entre mareas, sublitoral hasta $40 \mathrm{~m}$ (Snyder-Conn 1980).

Informes previos en CR: ninguno.

Distribución: de Baja California, Baja California Sur, Golfo de California, México a Perú (Lemaitre y Alvarez-Leon 1992, Haig y Harvey 1991, Hendrickx y Harvey 1999).

Comentario: primer informe para Costa Rica.

\section{Pagurus nanodes}

Haig \& Harvey, 1991

Material: ninguno.

Hábitat: desde intermareal hasta $3.5 \mathrm{~m}$ (Haig y Harvey 1991).

Informes previos en CR: bahía Brasilito y cerca de Puntarenas (Haig y Harvey 1991).

Distribución: de Costa Rica a Ecuador (Haig y Harvey 1991).

\section{Pagurus vetaultae}

Harvey \& McLaughlin, 1991

Material: ninguno.

Hábitat: sublitoral somero en sustratos de grava gruesa, de uno a $7 \mathrm{~m}$ (Harvey y McLaughlin 1991).

Informes previos en CR: al norte de bahía Santa Elena, Guanacaste y lado norte de isla del Caño, Puntarenas, Costa Rica (Harvey y McLaughlin 1991).

Distribución: del área de San CarlosGuaymas en el golfo de California, México a la bahía de Panamá (Harvey y McLaughlin 1991). 
Pagurus virgulatus

Haig \& Harvey, 1991

Material: ninguno.

Hábitat: de 5 a 40 m. (Haig y Harvey 1991)

Informes previos en CR: Puerto Parker(actualmente bahía San Elena, localidad tipo); bahía Brasilito; al suroeste de isla Pájara, bahía Weston y Bajo Alcyone, isla del Coco (Haig y Harvey 1991).

Distribución: de Acapulco, México al Ecuador e isla del Coco (Haig y Harvey 1991).

\section{Phimochirus californiensis \\ (Benedict, 1892)}

Material: ninguno.

Hábitat: litoral hasta $129 \mathrm{~m}$ de profundidad (McLaughlin 1981b).

Informes previos en CR: isla del Coco (Haig et al. 1970); mar afuera al sur de Viradores; isla del Caño; mar afuera de isla Nuez, isla del Coco (McLaughlin 1981b).

Distribución: desde Santa Catalina, California hasta Ecuador, incluyendo las islas Galápagos y del Coco (McLaughlin 1981b).

\section{Phimochirus roseus}

(Benedict, 1892)

Material: ninguno.

Hábitat: de la zona entre mareas a $3 \mathrm{~m}$ de profundidad (McLaughlin 1981b).

Informes previos en CR: al norte de bahía Jobo y bahía Huevos, Guanacaste (McLaughlin 1981b).

Distribución: de Baja California a Ecuador (McLaughlin 1981b).

\section{Phimochirus venutus \\ (Bouvier, 1898)}

Material: $1 \sigma^{\top}$, isla del Caño, 20.X.1984 (UCR 1071-01).

Hábitat: de 8-20 $\mathrm{m}$ de profundidad (McLaughlin 1981b).
Informes previos en CR: isla Solera, Quepos (McLaughlin 1981b).

Distribución: de Baja California hasta Ecuador (McLaughlin 1981b).

\section{Rhodochirus hirtimanus \\ (Faxon, 1893)}

Material: ninguno.

Hábitat: de 36-182 $\mathrm{m}$ de profundidad (McLaughlin 1981b).

Informes previos en CR: isla del Coco (Faxon 1895, McLauglin 1981b).

Distribución: golfo de California al norte de Perú incluyendo la isla del Coco y las islas Galápagos (McLaughlin 1981b).

Comentarios: localidad tipo es isla del Coco (McLaughlin 1981b).

\section{Tomopagurus merimaculosus}

(Glassell, 1937)

Material: 1, golfo de Nicoya, 11.IV.1980 (UCR 781-07). $10^{\pi}$, isla del Caño, 19.X.1964

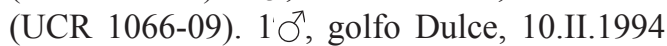
(UCR 2104-03). $3 \sigma^{\top}$, golfo de Nicoya, 13.II.1994 (UCR 2027-01).

Hábitat: de 35-183 m (McLaughlin 1981a).

Informes previos en CR: mar afuera de bahía Herradura y golfo Dulce (McLaughlin 1981a).

Distribución: desde el golfo de California hasta Colombia (McLaughlin 1981a).

\section{Tomopagurus purpuratus}

(Benedict, 1892)

Material: $4 O^{\top} 29$ (1 ov.), golfo Dulce. 12.XII.1993 (UCR 1995-03). 10َ, golfo Dulce, 18.II.1994 (UCR 2104-04).

Hábitat: de 36-274 m (McLaughlin 1981a).

Informes previos en CR: ninguno.

Distribución: desde el golfo de California a Colombia, incluyendo islas Revillagigedo y Galápagos (McLaughlin 1981a). 
Comentarios: primer informe para Costa Rica.

\author{
Xylopagurus cancellarius \\ Walton, 1950
}

Material: ninguno.

Hábitat: madera o en hoyos en juncos de Typha sp. a 73 m o más (Lemaitre 1989).

Informes previos en CR: bahía Herradura, Costa Rica (Lemaitre 1989).

Distribución: Pacífico Oriental Tropical: Costa Rica y Colombia (Lemaitre 1989).

Familia PARAPAGURIDAE Smith, 1882

\section{Parapagurus holtuisi \\ Lemaitre, 1989}

Material: ninguno.

Hábitat: a profundidades mayores a 200 m (Lemaitre y Alvarez-León 1992).

Informes previos en CR: informado para la isla del Coco como Pylopagurus pilosimanus abyssorum Henderson, 1888 por Faxon (1895) y renombrado como Pagurus holtuisi por Lemaitre (1989).

Distribución: de California a Chile (Lemaitre y Alvarez-León 1992), islas Galápagos (Hendrickx y Harvey 1999).

\section{Probeebei mirabilis}

Boone, 1926

Material: ninguno.

Hábitat: 1145-3995 m (Wicksten 1989).

Informes previos en CR: isla del Coco (Boone 1926).

Distribución: de Costa Rica a Perú (Wicksten 1989).

\section{DISCUSIÓN}

En este estudio y recopilación de información se indica la presencia de 114 especies de anomuros en aguas costarricenses. De estas, 20 especies son del Caribe, 96 son del Pacífico, y dos especies se encuentran en ambas costas. En 1993, Morán y Dittel informaron un total de 77 especies, con las especies informadas aquí la lista de anomuros conocidos para Costa Rica aumenta en 37 especies. De las 114 especies informadas, 29 son nuevos registros para el país, 15 del Caribe $(75 \%$ del total de especies del Caribe) y 14 del Pacífico (15\% del total de especies del Pacífico) (Cuadro 1). Dos especies son nuevos registros para la costa Caribe, pero no para el país, ya que habían sido previamente informadas para el Pacífico, $P$. armatus y $P$. galathinus.

Se amplia la distribución de seis especies hasta el Caribe de Costa Rica (Cuadro 1): $M$. flinti distribuida anteriormente en el golfo de México (Benedict 1902), al norte del arrecife Alacrán y mar afuera de Grenada (Chace 1942), se le amplía la distribución a la costa Caribe de Costa Rica. M. mortenseni distribuida en las islas Vírgenes, Panamá, costa Caribe de Colombia a Brasil (Gore 1982). M. poeyi: Florida, Antillas Mayores y Menores, costa norte de Suramérica y Panamá (Werding 1984). Se amplía la distribución de la especie $P$. cristobalensis desde ese mismo lugar a punta Mona. P. serratus: Antillas, Panamá y Colombia. A. paretii: de Carolina del Norte a Corpus Christi, Texas, a través de las Antillas Mayores a Santa Catarina, Brasil y en África Occidental de las islas Cabo Verde a Senegal y Ghana. P. diogenes: cabo Lookout, Carolina del Norte, a través del golfo de México y las Antillas Mayores a la isla de São Sevastiao, Brasil (Abele y Kim 1986).

Tres especies presentan ampliación de distribución en el Pacífico: M. gracilipes, conocida de Panamá a Colombia, ahora se presenta en Quepos; M. obesa conocida también del golfo de Panamá y Colombia se amplía al norte hasta el golfo de Nicoya; y N. bicapillatum conocida de Colombia y Ecuador se amplía al norte hasta punta Sucia, Garabito, Puntarenas.

Seis especies son informes nuevos para la Isla del Coco: A. elegans, P. biocellatus, $P$. glasselli, $P$. haigae, $P$. cancrisocialis y $P$. paguriconviva. De las especies de la isla del Coco cuatro especies son endémicas a la isla: $A$. rugosus, $P$. fecundus, E. janetae y $P$. cocoensis. 


\section{CUADRO 1}

Nuevos registros y ampliación de ámbito geográfico (de algunas especies) de anomuros para Costa Rica

\section{CARIBE}

Munida flinti

Megalobrachium mortenseni

Megalobrachium poeyi

Megalobrachium roseum

Megalobrachium soriatum

Pachycheles cristobalensis

Pachycheles pilosus

Pachycheles serratus

Petrolisthes armatus

Petrolisthes galathinus

Petrolisthes marginatus

Porcellana sayana

Albunea paretii

Calcinus tibicen

Petrochirus diogenes

Munida gracilipes

Munida obesa

Munidopsis hamata

Megalobrachium erosum

Megalobrachium festai

Megalobrachium garthi

Neopisosoma bicapillatum

Neopisosoma dohenyi

Pachycheles biocellatus

Petrolisthes glasselli

Petrolisthes haigae

Petrolisthes robsonae

Polyonyx nitidus

Albunea lucasia

Porcellana cancrisocialis

Porcellana paguriconviva

Aniculus elegans

Paguristes digueti

Pagurus lepidus

Tomopagurus purpuratus
Golfo de México y Grenada, a Limón

Islas Vírgenes, Panamá, costa Caribe de Colombia a Brasil, al Parque Nacional Cahuita Florida, Antillas Mayores y Menores, costa norte de Suramérica y Panamá, al Parque Nacional Cahuita

Nuevo registro para Costa Rica

Nuevo registro para Costa Rica

De una franja alrededor de la entrada Caribe del Canal de Panamá, a Punta Mona Nuevo registro para Costa Rica

Antillas, Panamá y Colombia, al Parque Nacional Cahuita

Nuevo registro para el Caribe de Costa Rica

Nuevo registro para el Caribe de Costa Rica

Nuevo registro para Costa Rica

Nuevo registro para Costa Rica

Carolina del Norte a Texas, de las Antillas Mayores a Santa Catarina, Brasil, de las islas Cabo Verde a Senegal y Ghana, a la Isla Uvita

Nuevo registro para Costa Rica

Carolina del Norte, al Golfo de México, las Antillas Mayores e isla de São Sevastiao, Brasil, a Manzanillo

\section{PACÍFICO}

Panamá y Colombia, a Quepos

Golfo de Panamá y Colombia, a Golfo de Nicoya

Nuevo registro para Costa Rica

Nuevo registro para Costa Rica

Nuevo registro para Costa Rica

Nuevo registro para Costa Rica

Puerto Utría, Colombia, La Libertad, Ecuador e islas Galápagos, a Puntarenas

Nuevo registro para Costa Rica

Se amplía distribución a la isla del Coco

Se amplía distribución a la isla del Coco

Se amplía distribución a la isla del Coco

Nuevo registro para Costa Rica

Nuevo registro para Costa Rica

Nuevo registro para Costa Rica

Se amplía distribución a la isla del Coco

Se amplía distribución a la isla del Coco

Se amplía distribución a la isla del Coco

Nuevo registro para Costa Rica

Nuevo registro para Costa Rica

Nuevo registro para Costa Rica 
De todas las especies informadas en la literatura y ahora en este estudio (114 especies), 30 especies, o sea el $26 \%$ del total no están presentes en las colecciones del Museo de Zoología.

El $75 \%$ del total de especies informadas aquí para el Caribe representan informes nuevos para Costa Rica. Este número tan alto se debe a lo poco estudiada que estaba la costa Caribe. Los informes nuevos para el Pacífico en esta recopilación, son 14 y representa el 15\% del total de especies, parecen pocas, pero si se considera que los anomuros del Pacífico han sido estudiados por muchos investigadores desde hace más de 100 años, el número de informes nuevos es relativamente alto (Cuadro 1). Estas dos observaciones nos llevan a concluir que si se profundizan los estudios en ambas costas es muy probable que encontremos, por un lado, más especies que no habían sido informadas para Costa Rica, como posiblemente, también especies nuevas para la Ciencia.

\section{AGRADECIMIENTOS}

Este trabajo fue posible gracias al apoyo de la Escuela de Biología, a través del Museo de Zoología, de la Vicerrectoría de Investigación, Universidad de Costa Rica (proyectos 808-96601), de la biblioteca de RSMAS, Universidad de Miami, y de innumerables colegas. Esta es una contribuión del Museo de Zoología, Escuela de Biología, Universidad de Costa Rica.

\section{RESUMEN}

El grupo de los cangrejos anomuros es uno de los mejor conocidos de la costa Pacífica de Costa Rica, pero muy poco conocidos de la costa Caribe. En esta recopilación, basada en la literatura y en las colecciones del Museo de Zoología, Escuela de Biología, Universidad de Costa Rica, informamos de la presencia de 114 especies del Infraorden Anomura en Costa Rica, 20 especies del Caribe, 96 especies del Pacífico, y dos especies presentes en ambas costa. Veintinueve especies son informes nuevos para Costa Rica, 15 del Caribe (75\% del total de especies informadas para esa costa) y 14 del Pacífica ( $15 \%$ del total de esa costa). La distribución de diez especies es ampliada hasta Costa Rica, siete en el Caribe y tres en el Pacífico.
Seis especies son informadas por primera vez para la Isla del Coco, donde además hay cuatro especies endémicas.

Palabras clave: Anomura, Crustacea, cangrejos, biodiversidad, Costa Rica.

\section{REFERENCIAS}

Abele, L.G. \& W. Kim. 1986. An illustrated guide to the marine decapods crustaceans of Florida. Dept. Environm. Reg. Tech. Series, Florida 8: 1-326.

Abele, L.G. \& W. Kim. 1989. The decapods crustaceans of the Panama Canal. Smithson. Contr. Zool. 482: 1-50.

Ball, E.E. 1972. Observation on the biology of the hermit crab, Coenobita compressus H. Milne Edwards (Decapoda, Anomura) on the west coast of the Americas. Rev. Biol. Trop. 20: 265-273.

Ball, E.E. \& J. Haig. 1974. Hermit crabs from the tropical eastern Pacific. I. Distribution color and natural history of some common shallow-water species. Bull. South. Cal. Acad. Sci. 73: 95-104.

Benedict, J.E. 1902. Description of a new genus and forty-six new species of crustaceans of the family Galatheidae, with a list of the known marine species. Proc. U.S. Nat. Mus. 26: 243-334.

Boone, L. 1926. A new family of Crustacea. Preliminary technical description. Zool. Soc. Bull. 29:73

Boone, L. 1930. Scientific results of the cruises of the yachts "Eagle" and "Ara", 1921-1928, William K. Vanderbilt, commanding. Crutacea: Anomura, Macrura, Schizopoda, Isopoda, Amphipoda, Mysidacea, Cirripedia and Copepoda. Bull. Vanderbilt Mar. Mus 3:1-221.

Boone, L. 1932. The littoral crustacean fauna of the Galapagos Islands. Part II. Anomura. Zoologica 14: $1-62$.

Bright, D.B. 1966. Land crabs of Costa Rica. Rev. Biol. Trop. 14: 183-203.

Brusca, R. C. 1980. Common Intertidal Invertebrates of the Gulf of California. Univ. Arizona, Tucson. 513 p.

Campos, N. \& R. Lemaitre. 1994. A new Calcinus (Decapoda: Anomura: Diogenidae) from the tropical western Atlantic and a comparison with other species of the genus from the region. Proc. Biol. Soc. Wash. 107: $137-150$.

Chace, F.A. 1942. Reports on the scientific results of the Atlantis Expedition to the West Indies, under the joint 
auspices of the University of Havana and Harvard University. Torreia 11: 1-105.

Chace, F.A. 1962. The non-brachyuran decapod crustaceans of Clipperton Island. Proc. U.S. Nat. Mus. 113: 605-635.

Dexter, D.M. 1974. Sandy-beach fauna of the Pacific and Atlantic coast of Costa Rica and Colombia. Rev. Biol. Trop. 22: 51-66.

Effort, I.E. 1971. The species of sand crabs in the genus Lepidopa (Decapoda: Albuneidae). Zool. Anz. Leipzig 186: 59-102.

Efford, I.E. 1973. The distribution of the sand crabs, Hippa sitrigillata (Stimpson) and Hippa pacifica (Dana) in the eastern Pacific Ocean (Decapoda: Anomura). Crustaceana 23: 119-122.

Efford, I.E. 1976. Distribution of the sand crabs in the genus Emerita (Decapoda: Hippidae). Crustaceana 30: 169-183.

Faxon, W. 1893. Preliminary descriptions of new species of Crustacea. Bull. Mus. Comp. Zool. 24: 149-220.

Faxon, W. 1895. Reports on an exploration off the west coast of México, Central and South America, and off the Galápagos Islands by the U.S. Fish Commission Steamer "Albatros", during 1891...XV. The stalkeyed Crustacea. Mem. Mus. Com. Zool. 18: 1-292.

García-Gómez, J. 1983. Revision of Iridopagurus (Crustacea, Decapoda, Paguridae) with the description of new species from American waters. Bull. Mar. Sci. 33: 10-54.

Gore, R.H. 1970. Pachycheles cristobalensis, sp. nov. with notes on the porcellanid crabs of the southern Caribbean. Bull. Mar. Sci. 20: 957-970.

Gore, R.H. 1974. On a small collection of porcellanid Crabs from the Caribbean Sea (Crustacea, Decapoda, Anomura). Bull. Mar. Sci. 24:700-721.

Gore, R.H. 1982. Porcellanidae crabs from the coasts of México and Central America (Crustacea, Decapoda, Anomura). Smithson. Contrib. Zool. 363: 1-34.

Gore R.H. \& L. G. Abele. 1973. Three new species of porcellanid crabs (Crustacea, Decapoda, Porcellanidae) from the Bay of Panama and adjacent Caribbean waters. Bull. Mar. Sci. 23: 559-573.

Gore, R.H. \& L.G. Abele. 1976. Shallow water porcelain crabs from the Pacific coast of Panama and adjacent Caribbean waters (Crustacea, Anomura, Porcellanidae). Smithson. Contrib. Zool. 237: 1-30.
Gordon, I. 1938. A comparison of the two genera Albunea and Lepidopa (Crustacea, Anomura), with description of a new species from Singapore. Bull. Raffles Mus. 14: 186-197.

Haig, J. 1956. The Galatheidea (Crustacea: Anomura) of the Allan Hancock Atlantic Expedition with review of the Porcellanidae of the Western North Atlantic. Rep. Allan Hancock Atlant. Exped. 8: 1-148.

Haig, J. 1957. Four new porcellain crabs from the eastern Pacific. Bull. South. Calif. Acad. Sci. 56: 31-41.

Haig, J. 1960. The Porcellanidae (Crustacea: Anomura) of the eastern Pacific. Allan Hancock Pac. Exped. 24: 1-440.

Haig, J. 1962. Papers from Dr. Th. Mortensen's Pacific expedition 1914-1916. LXXIX. Porcellanid crabs from eastern and western America. Vidensk. Meddel. Dansk Naturhist. Foren. Kjobenhavn 124: 171-192.

Haig, J. 1968. Eastern Pacific expeditions of the New York Zoological Society. Porcellanid crabs (Crustacea: Anomura) from the west coast of tropical America. Zoologia 53: 57-74.

Haig, J. 1974. Observations on the lithodid crabs of Peru, with description of two new species. Bull. South. Cal. Acad. Sci. 73: 152-164.

Haig, J. \& A.W. Harvey. 1991. Three new species of the Pagurus lepidus complex (Decapoda, Anomura, Paguridae) from the eastern Pacific. Contr. Sci, Los Angeles County Nat. Hist. Mus. 430: 1-11.

Haig, J. \& A.J. Provenzano. 1965. A new genus and two new species of diogenid hermit crabs (Decapoda, Anomura). Crustaceana 9: 199-207.

Haig, J., T.S. Hopkins \& T.B. Scandand. 1970. The shallow water anomuran crab fauna of southwestern Baja California, Mexico. Trans. San Diego Soc. Nat. Hist. 16: 14-31.

Harvey, A.W. 1998. Rediscovery and range extension of the Galápagos "Endemic" Pachycheles velerae (Decapoda: Anomura: Porcellanidae). J. Crust. Biol. 18: 746-752.

Harvey, A.W. \& P. McLaughlin. 1991. Two new hermit crabs of the genus Pagurus (Provenzanoi group)(Crustacea, Anomura, Paguridae) from the eastern Pacific, with notes on their ecology. Contr. Sci., Los Angeles County Nat. Hist. Mus. 425: 13-21.

Hendrickx, M.E. 1995. Anomuros, p. 539-564. In W. Fischer, F. Krupp, W. Schneider, C. Sommer, K.E. Carpenter \& U.H. Niem (eds.). Guía FAO para 
la identificación de especies para los fines de la pesca. Pacífico Centro-oriental. Vol. 1. Plantas e Invertebrados. FAO, Roma.

Hendrickx, M.E. \& A.W. Harvey. 1999. Checklist of anomuran crabs (Crustacea: Decapoda) from the eastern tropical Pacific. Belg. J. Zool. 129: 363-389.

Hendrickx, M.E., M. Demestre, A. Esparza-Haro \& J. Salgado-Barragán. 1997. Stomatopod and decapod crustaceans collected during the CEEMEX P5 and CEEMEX P7 cruises to the Gulf of Tehuantepec, México. Oceánides 11: 1-28.

Lemaitre, R. 1989. Revision of the genus Parapagurus (Anomura: Paguroidea: Parapaguridae), including redescription of the western Atlantic species. Zool. Verhand. 253: 1-106.

Lemaitre, R. \& R. Alvarez-León. 1992. Crustáceos decápodos del Pacífico Colombiano: Lista de especies y consideraciones zoogeográficas. An. Inst. Mar. Punta Betín 21: 33-76.

Lemaitre, R. \& P.A. McLaughlin. 1996. Revision of Pylopagurus and Tomopagurus (Crustacea: Anomura: Paguridae), with the description of new genera and species. Part V. Anisopagurus McLaughlin, Manucomplanus McLaughlin and Protoniopagurus new genus. Bull. Mar. Sci. 59: 89-141.

MacPherson, E. 1988. Revision of the family Lithodidae Samouelle, 1819 (Crustacea, Decapoda, Anomura) in the Atlantic Ocean. Monogr. Zool. Mar. (Instituto de Ciencias del Mar, Barcelona) 2: 9-153.

Maurer, D., C.E. Epifanio, H.K. Dean, S. Howe, J.A Vargas, A.I. Dittel \& M.M. Murillo. 1984. Benthic invertebrates of a tropical estuary: Gulf of Nicoya, Costa Rica. J. Nat. Hist. 18: 47-61.

McLaughlin, P.A. 1981a. Revision of Pylopagurus and Tomopagurus (Crustacea: Decapoda: Paguridae), with the descriptions of new genera and species: Part I. Ten new genera of the Parapaguridae and a redescription of Tomopagurus A. Milne Edwards and Bouvier. Bull. Mar. Sci. 31: 1-30.

McLaughlin, P.A. 1981b. Revision of Pylopagurus and Tomopagurus (Crustacea: Decapoda: Paguridae), with the descriptions of new genera and species: Part II. Rhodochirus McLaughlin and Phimochirus McLaughlin. Bull. Mar. Sci. 31: 329-365.

McLaughlin, P.A. 1982. Revision of Pylopagurus and Tomopagurus (Crustacea: Decapoda: Paguridae), with the descriptions of new genera and species: Part III. Agaricochirus McLaughlin, and Enallopaguropsis McLaughlin. Bull. Mar. Sci. 32: 823-855.

Moran, D.A. 1984. Additions to the known anomuran fauna of El Salvador, Central America (Crustacea: Decapoda). J. Crust. Biol. 4: 72-84.

Moran, D.A. \& A.I. Dittel. 1993. Anomuran and Brachyuran crabs of Costa Rica: annotated list of species. Rev. Biol. Trop. 41: 599-617.

Pequegnat, W.E. \& L.H. Pequegnat. 1973. Munidopsis albatrossae, a new species of deep-sea (Galatheidae, Decapoda, Anomura) from the eastern Pacific Ocean. Crustaceana 24: 163-168.

Pesta, O. 1931. Ergebnisse der Österreichischen Biologischen Costa Rica Expedition 1930. I. Teil. Crustacea Decapoda aus Costa Rica. Ann. Naturhist. Mus. Wien 45: 173-181.

Snyder-Conn, E. 1980. Arthropoda: Crustacea, Paguroidea and Coenobitoidea, p. 275-285. In R. Brusca (ed.). Common Intertidal Invertebrates of the Gulf of California. Univ. Arizona, Tucson.

Vargas, J. A., H.K. Dean, D. Maurer \& P. Orellana. 1985. Lista preliminar de invertebrados asociados a los sedimentos del Golfo de Nicoya, Costa Rica. Brenesia 24: 327-342.

Werding, B. 1977. Los porcelánidos (Crustacea: Anomura: Porcellanidae) de la región de Santa Marta, Colombia. An. Inst. Inv. Mar. Punta Betín 9: 173-214.

Werding, B. 1984. Porcelánidos (Crustacea: Anomura: Porcellanidae) de la isla de Providencia, Colombia. An. Inst. Inv. Mar. Punta Betín 14: 3-16.

Werding, B. \& J. Haig. 1982. The porcellanids crabs of Isla Gorgona, Pacific coast of Colombia, with a description of Clastotoechus gorgonensis sp. nov. (Crustacea: Anomura). An. Inst. Inv. Mar. Punta Betín 9: 173-214.

Wicksten, M.K. 1989. Ranges of offshore decapod crustaceans in the eastern Pacific Ocean. Trans. San Diego Soc. Nat. Hist. 21: 291-316.

Williams, A. 1984. Shrimps, lobsters and crabs of the Atlantic coast of the eastern United States, Maine to Florida. Smithson. Inst., Washington, D.C. 550 p.

Wolff, T. 1961. Animal life from a single abyssal trawling. Galathea Rep. 5: 129-162. 